\title{
Maternal adverse effects of different antenatal magnesium sulphate regimens for improving maternal and infant outcomes: a systematic review
}

Emily S Bain ${ }^{1 *}$, Philippa F Middleton ${ }^{1}$ and Caroline A Crowther ${ }^{1,2}$

\begin{abstract}
Background: Antenatal magnesium sulphate, widely used in obstetrics to improve maternal and infant outcomes, may be associated with adverse effects for the mother sufficient for treatment cessation. This systematic review aimed to quantify maternal adverse effects attributed to treatment, assess how adverse effects vary according to different regimens, and explore women's experiences with this treatment.

Methods: Bibliographic databases were searched from their inceptions to July 2012 for studies of any design that reported on maternal adverse effects associated with antenatal magnesium sulphate given to improve maternal or infant outcomes. Primary outcomes were life-threatening adverse effects of treatment (death, cardiac arrest, respiratory arrest). For randomised controlled trials, data were meta-analysed, and risk ratios (RR) pooled using fixed-effects or random-effects models. For non-randomised studies, data were tabulated by design, and presented as RR, odds ratios or percentages, and summarised narratively.
\end{abstract}

Results: A total of 143 publications were included (21 randomised trials, 15 non-randomised comparative studies, 32 case series and 75 reports of individual cases), of mixed methodological quality. Compared with placebo or no treatment, magnesium sulphate was not associated with an increased risk of maternal death, cardiac arrest or respiratory arrest. Magnesium sulphate significantly increased the risk of 'any adverse effects' overall (RR 4.62, 95\% Cl 2.42-8.83; 4 trials, 13,322 women), and treatment cessation due to adverse effects (RR 2.77; 95\% Cl 2.32-3.30; 5 trials, 13,666 women). Few subgroup differences were observed (between indications for use and treatment regimens). In one trial, a lower dose regimen ( $2 \mathrm{~g} / 3$ hours) compared with a higher dose regimen ( $5 \mathrm{~g} / 4$ hours) significantly reduced treatment cessation (RR 0.05; 95\% Cl 0.01-0.39, 126 women). Adverse effect estimates from studies of other designs largely supported data from randomised trials. Case reports supported an association between iatrogenic overdose of magnesium sulphate and life-threatening consequences.

Conclusions: Appropriate administration of antenatal magnesium sulphate was not shown to be associated with serious maternal adverse effects, though an increase in 'minor' adverse effects and treatment cessation was shown. Larger trials are needed to determine optimal regimens, achieving maximal effectiveness with minimal adverse effects, for each antenatal indication for use. Vigilance in the use of magnesium sulphate is essential for women's safety.

Keywords: Magnesium sulphate, Magnesium sulfate, Antenatal, Adverse effect, Systematic review

\footnotetext{
* Correspondence: emily.bain@adelaide.edu.au

${ }^{1}$ Australian Research Centre for Health of Women and Babies, Robinson

Institute, Discipline of Obstetrics and Gynaecology, School of Paediatrics and Reproductive Health, The University of Adelaide, 72 King William Road,

Adelaide, South Australia, Australia

Full list of author information is available at the end of the article
} 


\section{Background}

Magnesium sulphate has a long history of use in obstetrics. It is supported as the first line treatment for women with eclampsia [1-3], and is the drug of choice for women with severe pre-eclampsia [4]; it has been widely used as a tocolytic, however, benefit for this indication remains unproven $[5,6]$. Most recently antenatal magnesium sulphate has been supported for neuroprotection of the fetus, and it is thus now recommended for women at risk of very preterm birth [7].

Although life-threatening maternal adverse effects of magnesium sulphate are considered extremely rare in obstetrics [8], severe consequences of magnesium toxicity including respiratory arrest, cardiac arrest and death have been detailed in case reports. The 'well recognised' and more commonly reported maternal adverse effects of magnesium sulphate include flushing, increased warmth and sweating due to the peripheral vasodilatory effects of magnesium, and nausea, vomiting, headaches, muscle weakness, blurred vision, and intravenous (IV) or intramuscular (IM) site pain or discomfort [8]. Though such maternal adverse effects may be considered comparatively 'minor,' they have been associated with the need for early cessation of this therapy, which has benefits when used for maternal and fetal neuroprotection $[4,7]$.

While maternal adverse effects following antenatal magnesium sulphate administration are well known [4-7,9], the risk of individual events is unclear, and there has been a dearth of evidence regarding how such adverse effects vary by different regimens. Variation in aspects of the regimens such as the route of administration, dose, and duration, may help to explain differences in adverse effects of magnesium sulphate experienced among women receiving treatment. Although recent evidence suggests that on average, there are not large differences in the risk estimates of adverse effects from randomised controlled trials and observational studies [10], some uncertainty remains regarding the consistency of estimates provided by diverse study designs [11].

In view of the extremely widespread use of antenatal magnesium sulphate in obstetric practice, in this systematic review we aimed to quantify the extent of maternal adverse effects attributed to treatment, explore any variation in risk estimates between study designs, and to assess how such maternal adverse effects vary according to different regimens for administration and different indications for use. Implementation of this therapy may be strengthened, and the safety improved, if guidelines and recommendations for practice can be based on such knowledge. As it is known that maternal adverse effects may affect adherence and therapy cessation, we additionally aimed to explore and better understand women's responses to their experiences with this therapy.

\section{Methods}

\section{Search strategy}

Additional file 1 provides the PRISMA checklist. A comprehensive search of the bibliographic databases, MEDLINE, Embase, CENTRAL (Cochrane Central Register of Controlled Trials) and TOXLINE, was undertaken from their respective inceptions to July 2012, using a combination of $\mathrm{MeSH}$ and free text terms [12]. The search strategies used are given in Additional file 2. No date or language restrictions were applied, however, because of logistical constraints, for non-English papers only those with an available partial/full translation were retrieved. Conference Proceeding Citation Index-Science, OpenSIGLE, ClinicalTrials.gov, Current Controlled Trials metaRegister of Controlled Trials, International Clinical Trials Registry, and Google were additionally searched using key word searches (including to identify any publically available incident reports from patient safety organisations). The reference lists of any eligible articles identified were checked for additional references. The blog search engine blogsearch. google.com and the search engine Google, limited to Discussions, were searched using key words (["magnesium sulfate" OR "magnesium sulphate"] AND pregnancy) (sorted by relevance). Because of logistical constraints, it was pre-specified that sampling would cease once 20 relevant blogs and 10 relevant discussion forum threads were identified, with up to a total of five relevant threads from each original site sampled, if available.

\section{Inclusion criteria \\ Studies}

We included intervention (randomised, cluster-randomised, quasi-randomised and non-randomised comparative studies) and observational studies (cohort, case-control, crosssectional, case series and case reports). We included studies available as abstracts only, along with full-text publications. Personal blogs and discussion forum threads from pregnancy-related internet sites were included, along with incident reports from patient safety organisations.

\section{Participants, interventions and comparisons}

We included women given antenatal magnesium sulphate: for pre-eclampsia/eclampsia (including when it was continued/initiated in the immediate postpartum period); for tocolysis to women in preterm labour or who had had at least one episode of threatened preterm labour; for neuroprotection of the fetus, to women considered at risk of preterm birth (less than 37 weeks' gestation), or at term, regardless of the regimen for administration (including iatrogenic overdoses). We excluded studies where women were given oral magnesium sulphate, and where magnesium sulphate was given as an adjuvant during anaesthesia, or where magnesium sulphate was given in combination with another agent for tocolysis. We included instances 
where magnesium sulphate was compared to no placebo, placebo or to a different magnesium sulphate regimen, and/or, where the study's exposure was magnesium sulphate. We excluded studies where magnesium sulphate was compared to an alternative therapy.

\section{Outcomes}

We included studies that reported data on maternal adverse effects associated with magnesium sulphate. Primary outcomes were life-threatening adverse effects of treatment (death, cardiac arrest, respiratory arrest). Secondary outcomes included other maternal-reported or clinical maternal adverse effects attributed to treatment (e.g. warmth and flushing, arm discomfort), outcomes associated with interventions to reduce potential/ actual adverse effects (e.g. use of calcium gluconate, discontinuation of treatment), and other outcomes of interest (including caesarean section, pulmonary oedema, and postpartum haemorrhage). We used the definitions as used by the study authors.

\section{Study selection}

After screening all titles and abstracts, we obtained the fulltext article for any study which appeared to meet the inclusion criteria based on the title and/or abstract, along with any reviews that may have provided relevant references. All full-text articles and abstracts were assessed for inclusion. Each stage was carried out by one reviewer (ESB) with the second reviewer (PFM) assessing a random sample (10\% of the total). We resolved any discrepancies through discussion, or if required, we consulted the third reviewer (CAC).

\section{Data extraction and management}

Once a study was included, data were extracted using a standardised form. Data extracted included information regarding study design, participants, the magnesium sulphate regimen(s), the control/comparison if applicable, maternal adverse effects reported and results relevant to the review, the risk of bias, confounding and relevance. For personal blogs and discussion forum threads, information regarding perceived purpose and/or benefits of treatment, and women's experiences with treatment, particularly considering adverse effects were extracted. Extraction was carried out by one reviewer (ESB), with the second reviewer (PFM) independently extracting a random sample (10\% of the total, and all included randomised controlled trials). We resolved any discrepancies through discussion, or if required, we consulted the third reviewer (CAC).

\section{Assessment of study quality/ risk of bias}

Quality appraisal of intervention studies was undertaken utilising established guidelines provided in the Cochrane Handbook for Systematic Reviews of Interventions [13]. The quality assessment of observational studies was guided by recommendations from the Cochrane Handbook on assessing the quality of non-randomised studies [13] (which highlights that attention must be paid particularly to selection bias) and principles of the Newcastle-Ottawa Scale [14], where we judged the quality of each study on three main aspects: the selection of the study groups; the comparability of the groups; and the ascertainment of either the exposure of outcome of interest for case-control or cohort studies respectively; for case series we primarily considered selection of the study group. As it has been suggested that the quality of adverse effect detection and reporting is not always adequately assessed, it was also important to consider the methods used to detect adverse effects and how rigorous these methods were, along with an assessment of incomplete reporting $[12,15]$.

\section{Data synthesis and analysis}

The analysis and presentation of results were categorised by study design. Statistical analyses were performed using Review Manager, version 5.1 (The Cochrane Collaboration, Copenhagen, Denmark).

For intervention studies we presented quantitative data from individual studies where possible as risk ratios (RR) with 95\% confidence intervals (CI) for dichotomous outcomes. For all outcomes, we carried out analyses as far as possible on an intention-to-treat basis. Pooled estimates (summary RR with 95\% CI) were calculated using fixed-effect meta-analysis (Mantel-Haenszel method) where there was a sufficient quantity of data, with clinical homogeneity. Where we considered that there was clinical heterogeneity sufficient to expect the underlying effects differed between trials, or there was substantial statistical heterogeneity (where $\mathrm{I}^{2}$ was greater than $30 \%$ and either $\mathrm{T}^{2}$ was greater than zero, or there was a low P-value (less than 0.10) in the $\mathrm{Chi}^{2}$ test), summary estimates were calculated using random-effects meta-analysis.

Separate comparisons were performed for those studies assessing magnesium sulphate versus no treatment/placebo, and those comparing different magnesium sulphate regimens. For all review outcomes, we conducted subgroup analyses based on indication for use (i.e. given for preeclampsia/eclampsia; fetal neuroprotection; tocolysis), as this was considered likely to influence outcomes. Additional subgroup analyses were planned if sufficient data were available based on aspects of the magnesium sulphate regimen (i.e. route of administration; dose). We assessed subgroup differences by interaction tests available within Review Manager, and where applicable, we have quoted the $\mathrm{Chi}^{2}$ statistic and P-value, and the interaction test $\mathrm{I}^{2}$ value. We included only primary outcomes and the outcomes: discontinuation due to adverse effects, calcium gluconate use, and 'any adverse effects' in subgroup analyses.

For observational studies (cohort, case-control, crosssectional, case series) we presented effect estimates where 
possible as percentages, RR or odds ratios (OR) with $95 \%$ CIs, adjusted RR or OR if reported with $95 \%$ CIs, or P-values only, in tabular format based on study type; we used narrative synthesis to summarise the studies. Data from case reports were tabulated and subsequently grouped according to themes. For personal blogs and discussion forum threads, relevant text was tabulated (considering perceived purpose/benefits of magnesium sulphate and experience of magnesium sulphate therapy, before, during and after treatment), and thematic analysis techniques were used to identify and summarise emerging themes.

\section{Results}

\section{Study selection}

The results of the search strategy, including the sources of the studies, culling and final inclusion of studies are shown in Figure 1. The initial database searching identified 5,062 articles. Review of the abstracts/titles and exclusion of irrelevant/duplicate articles yielded 1,034 articles. Of these articles, we excluded 896 for the documented reasons. We therefore included 138 studies, along with an additional five studies identified through other searching; a total of 143 studies (see Additional file 3 for References to all included reports). In the case of multiple publications from the same study, we included the report with the most relevant data relating to adverse effects.

\section{Evidence from randomised controlled trials}

Twenty one randomised trials (16,812 women) were included, the characteristics of which are detailed in

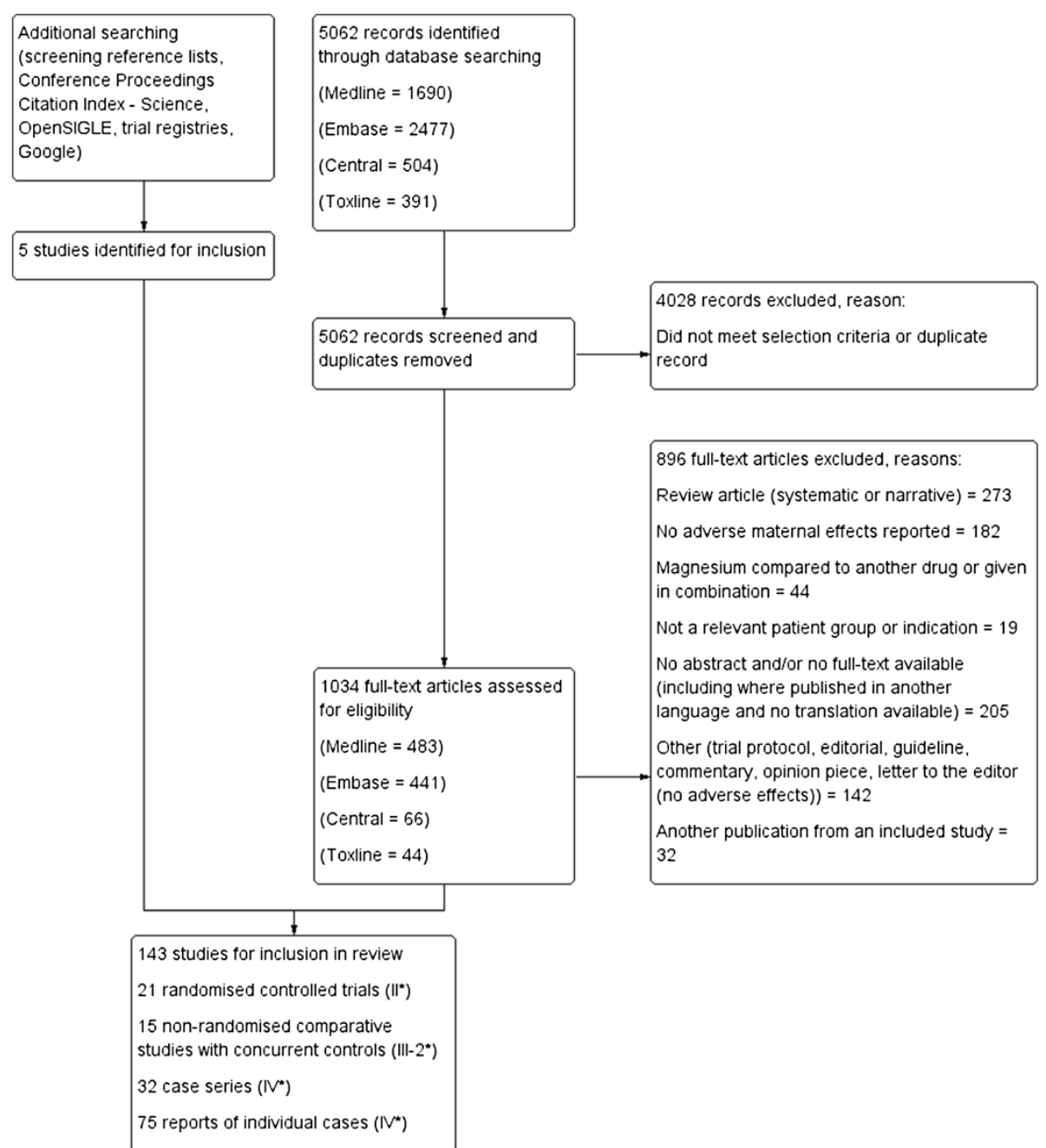

Figure 1 Flow diagram of included studies. *Numbers indicate level of evidence, according to Australian Government National Health and Medical Research Council (NHMRC) Evidence Hierarchy Available at: https:/www.nhmrc.gov.au/_files_nhmrc/file/guidelines/developers/nhmrc_levels_grades_ evidence_120423.pdf. 
Additional file 4, and the risk of bias assessment presented in Figures 2, 3 and 4. The trials assessed a variety of different treatment regimens with varying comparators, and are therefore assessed under six different comparisons:

1. Magnesium sulphate versus placebo or no treatment (11 trials).

2. Lower dose versus higher dose magnesium sulphate IM maintenance (2 trials).

3. Magnesium sulphate IV maintenance versus IM maintenance (3 trials).

4. Short versus standard (24 hour) postpartum magnesium sulphate maintenance (2 trials).

5. Lower dose versus higher dose magnesium sulphate IV maintenance (2 trials).

6. 'Ready-to-use' magnesium sulphate solution versus a reference drug requiring dilution (1 trial).

Considering the risk of bias for trials in Comparison 1 (magnesium sulphate versus placebo or no treatment), sequence generation and allocation concealment were adequate in the majority of trials $(6 / 11$ and $8 / 11$ trials respectively) (Figure 2). For other trials, it was unclear whether this was adequate, and for one trial, allocation concealment was not considered adequate. For six trials, blinding of personnel, women and outcome assessors was considered adequate, whilst for four trials this was not considered adequate, and for one trial this was unclear. The randomised trials in Comparisons 2-6 (different magnesium sulphate regimens) were considered at a comparatively higher risk of bias overall (Figure 3). For the majority of trials, it was unclear whether sequence generation and allocation concealment were adequate (5/10 and 8/10 trials respectively). Blinding of personnel and women was not possible in any of the trials; none of the trials reported that outcomes were assessed in a blinded manner.

\section{Comparison 1: magnesium sulphate versus placebo or} no treatment

This comparison included 11 trials with 15,709 women [16-26]. In six trials, the indication for use of magnesium sulphate was the prevention or treatment of eclampsia; for three trials, the indication was fetal neuroprotection, and for two trials, the prevention of preterm birth (see Table 1 and Additional file 5 for effect estimates and forest plots for outcomes in Comparison 1).

\section{Life-threatening adverse effects of treatment}

No significant differences were seen between magnesium sulphate and placebo/no treatment for maternal death (RR 0.53 ; $95 \%$ CI 0.26 to $1.09 ; 5$ trials, 14,662 women; Analysis 1.1), cardiac arrest (RR $0.80 ; 95 \%$ CI 0.21 to 2.98; 4 trials, 13,977 women; Analysis 1.2 ) or respiratory arrest (RR 2.50; 95\% CI 0.49 to $12.88 ; 4$ trials 13,977 women; Analysis 1.3).

\section{Interventions to limit adverse effects}

Women receiving magnesium sulphate experienced a significantly increased (almost three times) risk of discontinuing treatment due to associated adverse effects (RR 2.77; 95\% CI 2.32 to 3.30; 5 trials 13,666 women; Analysis 1.4). There were no significant differences between groups in the outcomes calcium gluconate administration (RR 1.35; 95\% CI 0.63 to $2.88 ; 2$ trials, 10,795 women; Analysis 1.5) and intensive care unit admission (RR 0.97; 95\% CI 0.72 to $1.30 ; 2$ trials, 11,172 women; Analysis 1.6).

\section{Adverse effects associated with treatment}

Women receiving magnesium sulphate were almost five times more likely to experience 'any side effects' in the four included trials (RR 4.62; 95\% CI 2.42 to 8.83; 13,322 women; Analysis 1.7). Women receiving magnesium sulphate compared with women receiving no treatment/placebo experienced an approximately 50\% increased risk of hypotension (RR 1.52; 95\% CI 1.10 to 2.11; 3 trials, 1,782 women; Analysis 1.11) and tachycardia (RR 1.53; 95\% CI 1.03 to $2.29 ; 1$ trial, 1,062 women; Analysis 1.12). Compared with women receiving no treatment/placebo, women receiving magnesium sulphate experienced an approximately $50 \%$ increased risk of problems at the IM injection site (RR 1.49; 95\% CI 1.25 to $1.79 ; 1$ trial, 4,553 women; Analysis 1.25), and more

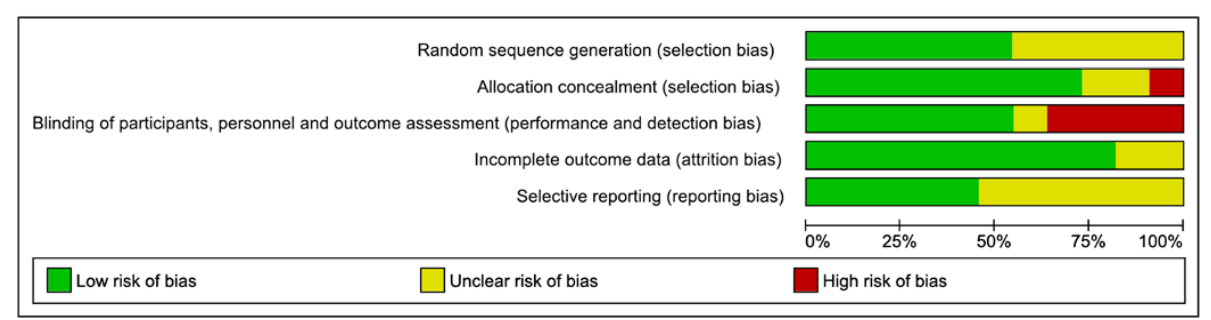

Figure 2 Risk of bias for randomised controlled trials (Comparison 1). Risk of bias graph showing review authors' judgements about each risk of bias item presented as percentages across included studies from Comparison 1. 


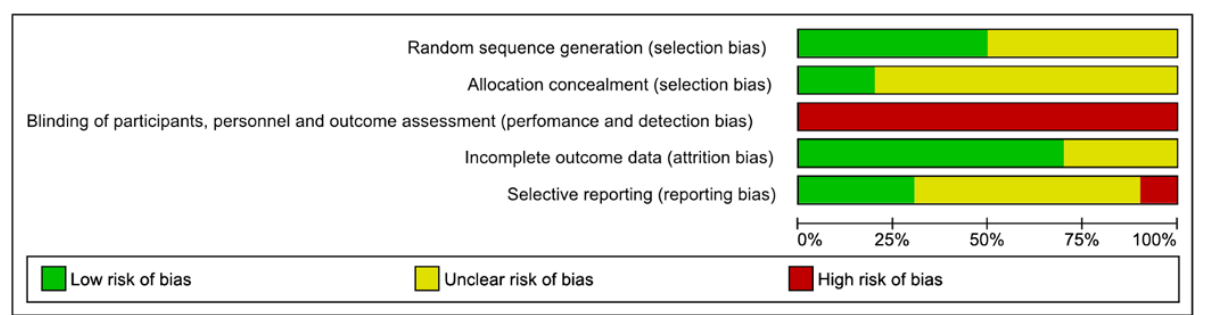

Figure 3 Risk of bias for randomised controlled trials (Comparisons 2-6). Risk of bias graph showing review authors' judgements about each risk of bias item presented as percentages across included studies from Comparisons 2-6.

than six times the risk of problems/discomfort at the IV site (RR 6.34; 95\% CI 3.10 to $12.98 ; 3$ trials, 8,704 women; Analysis 1.24).

Women receiving magnesium sulphate had an approximately $50 \%$ increased risk of respiratory depression (RR 1.41; 95\% CI 1.07 to 1.86 ; 5 trials, 14,098 women; Analysis 1.8), more than two times the risk of drowsiness/ confusion (RR 2.46; 95\% CI 1.83 to 3.29; 3 trials, 11,189 women; Analysis 1.16), headache (RR 2.21; 95\% CI 1.27 to 3.86; 2 trials, 10,556 women; Analysis 1.17), dizziness (RR 2.62; $95 \%$ CI 1.63 to 4.21 ; 2 trials, 11,054 women; Analysis 1.19), mouth dryness or thirst (RR 2.38; $95 \%$ CI 1.59 to 3.56; 2 trials, 11,054 women; Analysis 1.18) and blurred vision (RR 2.34; 95\% CI 1.32 to $4.14 ; 1$ trial, 1,062 women; Analysis 1.22), more than five times the risk of nausea and/or vomiting (RR 5.50; 95\% CI 2.29 to 13.22; 4 trials; 13,821 women; Analysis 1.14), nearly seven times the risk of flushing and warmth (RR 6.94; 95\% CI 4.19 to 11.49 ; 5 trials, 13,956 women; Analysis 1.13) and sweating (RR 6.37; 95\% CI 1.96 to 20.65; 2 trials, 3,265 women; Analysis 1.20), nearly 15 times the risk of itching and tingling (RR 14.98; 95\% CI 1.98 to 113.38 ; 1 trial, 9,992 women; Analysis 1.21), and more than 15 times the risk of muscle weakness (RR 15.81; 95\% CI 7.36 to 33.96; 3 trials, 10,212 women; Analysis 1.15).

There were no significant differences between groups for the outcomes absent/reduced tendon reflexes (RR 1.01; $95 \%$ CI 0.71 to 1.44 ; 3 trials; 11,241 women; Analysis 1.9 ) and slurred speech (RR 3.04; $95 \%$ CI 0.13 to 73.42; 1 trial, 135 women; Analysis 1.23).

\section{Other outcomes}

For women receiving magnesium sulphate compared to no treatment/placebo, a small significant increased risk of caesarean section was shown (RR 1.04; 95\% CI 1.00 to 1.08 ; 10 trials, 14,105 women; Analysis 1.26). No differences were seen between groups for the outcomes postpartum haemorrhage (RR 0.94; 95\% CI 0.87 to 1.04; 4 trials, 10,535 women; Analysis 1.27) and pulmonary oedema (RR 1.12; 95\% CI 0.72 to 1.74 ; 4 trials, 12,787 women; Analysis 1.28).

\section{Subgroup analysis by indication for use}

When considering indication for use, the subgroup interaction tests for the majority of outcomes were nonsignificant, indicating no differential effects according to the different reasons for administration (see Table 1 for effect estimates for indication for use subgroups and Additional file 5 for forest plots). While substantial statistical heterogeneity $\left(\mathrm{I}^{2}>90 \%\right)$ was observed for the outcomes 'any side effects', flushing and/or warmth and nausea and/or vomiting, this could not be explained by considering the indication for use of treatment. In each case the test for subgroup differences was non-significant ('any side effects': $\mathrm{Chi}^{2}=1.68, \mathrm{P}=0.43, \mathrm{I}^{2}=0 \%$; Analysis 1.7) (flushing and/or warmth: $\mathrm{Chi}^{2}=0.07, \mathrm{P}=0.79$, $\mathrm{I}^{2}=0 \%$; Analysis 1.13) (nausea and/or vomiting: $\mathrm{Chi}^{2}=$ $1.16, \mathrm{P}=0.28, \mathrm{I}^{2}=13.9 \%$; Analysis 1.14 ).

For the outcome problems at the IV site and/or arm discomfort, the subgroup interaction test indicated a significant difference between indication for use subgroups, and a possible differential effect in favour of receiving treatment for pre-eclampsia, with women receiving treatment for fetal neuroprotection being more likely to experience arm discomfort $\left(\mathrm{Chi}^{2}=25.80, \mathrm{P}=<0.00001\right.$, $\mathrm{I}^{2}=96.1 \%$; Analysis 1.24). It is possible, however, that the methods used to collect information on arm discomfort/ problems at the IV site differed substantially between trials, and could help to explain this observed differential effect.

\section{Subgroup analysis by regimen for administration}

To explore the effect of aspects of the regimen for administration of magnesium sulphate on adverse effects, the trials from Comparison 1 were grouped as prespecified where possible according to their dosage and/or route of administration (loading dose only; loading plus maintenance dose; $4 \mathrm{~g}$ IV loading dose plus any maintenance; 5-6 g IV loading dose plus any maintenance; $1 \mathrm{~g} /$ hour IV maintenance; $2-3 \mathrm{~g} /$ hour IV maintenance; IM maintenance).

For the outcomes maternal death, cardiac arrest, respiratory arrest and use of calcium gluconate, no significant differences were shown between the magnesium 
Figure 4 Risk of bias for randomised controlled trials

(Comparisons 1-6). Risk of bias summary showing review authors' judgements about each risk of bias item for included studies from Comparisons 1-6. Each risk of bias item is judged as at a low risk of bias, unclear risk of bias or high risk of bias.

sulphate and no magnesium sulphate groups for any of the subgroups, and the subgroup interaction tests for all outcomes indicated no significant differential effects across the treatment subgroups (see Table 1 for effect estimates for regimen subgroups and Additional file 5 for forest plots). The significantly increased risk of discontinuing treatment due to adverse effects and experiencing 'any side effects' for the magnesium sulphate group was seen across all of the different regimen subgroups (see Analyses 1.4 and 1.7); for both outcomes, the subgroup interaction tests did not indicate differential effects according to the subgroups.

\section{Comparison 2: lower dose versus higher dose IM} maintenance: prevention or treatment of eclampsia

This comparison included two trials with 176 women with both trials assessing magnesium sulphate for eclampsia, or 'imminent eclampsia' (see Table 2 and Additional file 5 for effect estimates and forest plots for Comparison 2). One trial compared a lower dose 'Dhaka' regimen from Bangladesh: $4 \mathrm{~g}$ IV and $6 \mathrm{~g}$ IM as a loading dose, and $2.5 \mathrm{~g}$ IM every four hours as maintenance, with a higher dose 'Bhalla' regimen: $4 \mathrm{~g}$ IV and $8 \mathrm{~g}$ IM as a loading dose, and $4 \mathrm{~g}$ IM every four hours as maintenance [27]. The second trial compared a loading dose of $4 \mathrm{~g}$ IV, and $2 \mathrm{~g}$ IM every three hours as maintenance, with Pritchard's regimen (a loading dose of $4 \mathrm{~g}$ IV and $10 \mathrm{~g} \mathrm{IM}$, and $5 \mathrm{~g}$ IM every four hours as maintenance) [28].

\section{Life-threatening adverse effects of treatment}

No significant difference between groups was shown for the risk of 'maternal death due to toxicity' in one trial of 126 women (RR 0.25, 95\% CI 0.01 to 6.05; Analysis 2.1). No other primary review outcomes were reported.

\section{Interventions to limit adverse effects}

Women allocated to the lower dose regimen were significantly less likely to have treatment stopped due to 'toxicity' in one trial, an approximate 95\% relative risk reduction (RR 0.05; 95\% 0.01 to 0.39; 126 women; Analysis 2.2). Women allocated to the lower dose regimen were significantly less likely to have a maintenance dose deferred or skipped due to adverse effects, an approximate $64 \%$ relative risk reduction (RR 0.36 ; $95 \%$ CI 0.20 to 0.63 ; 2 trials, 176 women; Analysis 2.3). No clear difference was shown for the need for calcium gluconate in one trial (RR 0.25 ; $95 \%$ CI 0.06 to 1.06 ; 1 trial, 50 women; Analysis 2.4). 
Table 1 Adverse effect estimates from randomised controlled trials (Comparison 1)

\begin{tabular}{|c|c|c|c|c|}
\hline Outcome or subgroup & Studies & Participants & Method $\left(I^{2}(\%)\right)^{*}$ & RR $(95 \% \mathrm{Cl})$ \\
\hline \multicolumn{5}{|c|}{ Comparison 1: Magnesium sulphate versus placebo or no treatment } \\
\hline 1.1 Death & $5[17,19,20,23,25]$ & 14662 & $F(0)$ & $0.53(0.26,1.09)$ \\
\hline 1.1.1 Treatment of pre-eclampsia/eclampsia & $2[17,20]$ & 10795 & $F(0)$ & $0.53(0.26,1.09)$ \\
\hline 1.1.2 Fetal neuroprotection & $3[19,23,25]$ & 3867 & $\mathrm{~F}(\mathrm{NA})$ & No deaths \\
\hline 1.1.3 LD only & $1[23]$ & 564 & $\mathrm{~F}(\mathrm{NA})$ & No deaths \\
\hline 1.1.4 LD and MD & $4[17,19,20,25]$ & 14098 & $F(0)$ & $0.53(0.26,1.09)$ \\
\hline 1.1.5 $4 \mathrm{~g} \mathrm{IV} \mathrm{LD} \mathrm{and} \mathrm{MD}$ & $3[17,19,20]$ & 11857 & $F(0)$ & $0.53(0.26,1.09)$ \\
\hline 1.1.6 5-6 g IV LD and MD & $1[25]$ & 2241 & $\mathrm{~F}(\mathrm{NA})$ & No deaths \\
\hline 1.1.7 $1 \mathrm{~g} /$ hour IV MD & $3[17,19,20]$ & 7264 & $F(0)$ & $0.41(0.12,1.43)$ \\
\hline 1.1.8 2-3 g/hour IV MD & $1[25]$ & 2241 & $\mathrm{~F}(\mathrm{NA})$ & No deaths \\
\hline 1.1.9 IM MD & $1[20]$ & 4593 & $\mathrm{~F}(\mathrm{NA})$ & $0.61(0.25,1.48)$ \\
\hline 1.2 Cardiac arrest & $4[19,20,23,25]$ & 13977 & $\mathrm{~F}(\mathrm{NA})$ & $0.80(0.21,2.98)$ \\
\hline 1.2.1 Treatment of pre-eclampsia/eclampsia & $1[20]$ & 10110 & $F(N A)$ & $0.80(0.21,2.98)$ \\
\hline 1.2.2 Fetal neuroprotection & $3[19,23,25]$ & 3867 & $\mathrm{~F}(\mathrm{NA})$ & No cardiac arrests \\
\hline 1.2.3 LD only & $1[23]$ & 564 & $F(N A)$ & No cardiac arrests \\
\hline 1.2.4 LD and MD & $3[19,20,25]$ & 13413 & $\mathrm{~F}(\mathrm{NA})$ & $0.80(0.21,2.98)$ \\
\hline 1.3 Respiratory arrest & $4[19,20,23,25]$ & 13977 & $\mathrm{~F}(\mathrm{NA})$ & $2.50(0.49,12.88)$ \\
\hline 1.3.1 Treatment of pre-eclampsia/eclampsia & $1[20]$ & 10110 & $\mathrm{~F}(\mathrm{NA})$ & $2.50(0.49,12.88)$ \\
\hline 1.3.2 Fetal neuroprotection & $3[19,23,25]$ & 3867 & $\mathrm{~F}(\mathrm{NA})$ & No respiratory arrests \\
\hline 1.3.3 LD only & $1[23]$ & 564 & $\mathrm{~F}(\mathrm{NA})$ & No respiratory arrests \\
\hline 1.3.4 LD and MD & $3[19,20,25]$ & 13413 & $\mathrm{~F}(\mathrm{NA})$ & $2.50(0.49,12.88)$ \\
\hline 1.4 Discontinuation due to adverse effects & $5[18-20,25,26]$ & 13666 & $F(0)$ & $2.77(2.32,3.30)$ \\
\hline 1.4.1 Treatment of pre-eclampsia/eclampsia & $2[20,26]$ & 10245 & $F(0)$ & $2.69(2.18,3.31)$ \\
\hline 1.4.2 Fetal neuroprotection & $2[19,25]$ & 3265 & $F(0)$ & $2.81(2.01,3.93)$ \\
\hline 1.4.3 Tocolysis & $1[18]$ & 156 & $\mathrm{~F}(\mathrm{NA})$ & $17.88(1.05,304.57)$ \\
\hline 1.4.4 LD and MD & $5[18-20,25,26]$ & 13666 & $F(0)$ & $2.77(2.32,3.30)$ \\
\hline 1.4.5 $4 \mathrm{~g} \mathrm{IV} \mathrm{LD} \mathrm{and} \mathrm{MD}$ & 3 [18-20] & 11328 & $F(0)$ & $2.75(2.28,3.31)$ \\
\hline 1.4.6 5-6 g IV LD and MD & $2[25,26]$ & 2338 & $F(0)$ & $2.94(1.69,5.12)$ \\
\hline 1.4.7 $1 \mathrm{~g} /$ hour IV MD & 1 [19] & 1062 & $\mathrm{~F}(\mathrm{NA})$ & $2.74(1.81,4.15)$ \\
\hline 1.4.8 2-3 g/hour IV MD & $3[18,25,26]$ & 2494 & $F(0)$ & $3.38(1.97,5.78)$ \\
\hline \multicolumn{5}{|l|}{ 1.5 Given calcium gluconate } \\
\hline 1.5.1 Treatment of pre-eclampsia/eclampsia & $2[17,20]$ & 10795 & $F(0)$ & $1.35(0.63,2.88)$ \\
\hline 1.5.2 $4 \mathrm{~g} \mathrm{IV} \mathrm{LD} \mathrm{and} \mathrm{MD}$ & $2[17,20]$ & 10795 & $F(0)$ & $1.35(0.63,2.88)$ \\
\hline 1.6 Intensive care unit admission & $2[19,20]$ & 11172 & $\mathrm{~F}(\mathrm{NA})$ & $0.97(0.72,1.30)$ \\
\hline 1.6.1 Treatment of pre-eclampsia/eclampsia & $1[20]$ & 10110 & $\mathrm{~F}(\mathrm{NA})$ & $0.97(0.72,1.30)$ \\
\hline 1.6.2 Fetal neuroprotection & 1 [19] & 1062 & $\mathrm{~F}(\mathrm{NA})$ & No admissions \\
\hline 1.7 Any side effects & $4[19,20,22,25]$ & 13322 & $\mathrm{R}(98)$ & $4.62(2.42,8.83)$ \\
\hline 1.7.1 Treatment of pre-eclampsia/eclampsia & $1[20]$ & 9992 & $\mathrm{~F}(\mathrm{NA})$ & $5.26(4.59,6.03)$ \\
\hline 1.7.2 Fetal neuroprotection & $2[19,25]$ & 3265 & R (98) & $3.82(1.38,10.59)$ \\
\hline 1.7.3 Tocolysis & $1[22]$ & 65 & $F(N A)$ & $26.71(1.64,435.03)$ \\
\hline 1.7.4 LD and MD & $4[19,20,22,25]$ & 13322 & R (98) & $4.62(2.42,8.83)$ \\
\hline 1.7.5 $4 \mathrm{~g} \mathrm{IV} \mathrm{LD} \mathrm{and} \mathrm{MD}$ & $2[19,20]$ & 11054 & R (99) & $3.52(1.49,8.32)$ \\
\hline 1.7.6 5-6 g IV LD and MD & $2[22,25]$ & 2268 & $F(5)$ & $6.28(5.36,7.35)$ \\
\hline 1.7.7 $1 \mathrm{~g} /$ hour IV MD & $2[19,20]$ & 6501 & R (98) & $3.31(1.59,6.88)$ \\
\hline
\end{tabular}


Table 1 Adverse effect estimates from randomised controlled trials (Comparison 1) (Continued)

\begin{tabular}{|c|c|c|c|c|}
\hline $1.7 .82-3 \mathrm{~g} /$ hour IV MD & $2[22,25]$ & 2268 & $F(5)$ & $6.28(5.36,7.35)$ \\
\hline 1.7.9 IM MD & $1[20]$ & 4553 & $\mathrm{~F}(\mathrm{NA})$ & $5.84(4.80,7.09)$ \\
\hline 1.8 Respiratory depression/other respiratory problems & $5[17-20,25]$ & 14098 & $F(29)$ & $1.41(1.07,1.86)$ \\
\hline 1.8.1 Treatment of pre-eclampsia/eclampsia & $2[17,20]$ & 10677 & $F(0)$ & $1.98(1.24,3.15)$ \\
\hline 1.8.2 Fetal neuroprotection & $2[19,25]$ & 3265 & $F(29)$ & $1.12(0.79,1.59)$ \\
\hline 1.8.3 Tocolysis & $1[18]$ & 156 & $\mathrm{~F}(\mathrm{NA})$ & $3.16(0.13,76.30)$ \\
\hline 1.9 Absent or reduced tendon reflexes & $3[17,20,23]$ & 11241 & $F(0)$ & $1.01(0.71,1.44)$ \\
\hline 1.9.1 Treatment of pre-eclampsia/eclampsia & $2[17,20]$ & 10677 & $F(0)$ & $1.00(0.70,1.42)$ \\
\hline 1.9.2 Fetal neuroprotection & $1[23]$ & 564 & $\mathrm{~F}(\mathrm{NA})$ & $1.94(0.18,21.32)$ \\
\hline \multicolumn{5}{|l|}{ 1.10 Respiratory depression and absent reflexes } \\
\hline 1.10.1 Treatment of pre-eclampsia/eclampsia & $3[17,20,21]$ & 10899 & $F(0)$ & $5.96(0.72,49.40)$ \\
\hline 1.11 Hypotension & $3[18,19,23]$ & 1782 & $F(0)$ & $1.52(1.10,2.11)$ \\
\hline 1.11.1 Fetal neuroprotection & $2[19,23]$ & 1626 & $F(0)$ & $1.51(1.09,2.09)$ \\
\hline 1.11.2 Tocolysis & $1[18]$ & 156 & $\mathrm{~F}(\mathrm{NA})$ & $3.16(0.13,76.30)$ \\
\hline \multicolumn{5}{|l|}{ 1.12 Tachycardia } \\
\hline 1.12.1 Fetal neuroprotection & $1[19]$ & 1062 & $F(N A)$ & $1.53(1.03,2.29)$ \\
\hline 1.13 Flushing and/or warmth & $5[19,20,23,25,26]$ & 13956 & $\mathrm{R}(92)$ & $6.94(4.19,11.49)$ \\
\hline 1.13.1 Treatment of pre-eclampsia/eclampsia & $2[20,26]$ & 10127 & $\mathrm{R}(91)$ & $6.39(2.44,16.74)$ \\
\hline 1.13.2 Fetal neuroprotection & $3[19,23,25]$ & 3829 & $\mathrm{R}(94)$ & $7.55(3.39,16.85)$ \\
\hline 1.14 Nausea and/or vomiting & $4[19,20,23,25]$ & 13821 & $R(92)$ & $5.50(2.29,13.22)$ \\
\hline 1.14.1 Treatment of pre-eclampsia/eclampsia & $1[20]$ & 9992 & $F(N A)$ & $8.88(5.46,14.43)$ \\
\hline 1.14.2 Fetal neuroprotection & $3[19,23,25]$ & 3829 & $\mathrm{R}(92)$ & $4.60(1.54,13.73)$ \\
\hline 1.15 Muscle weakness & $3[16,18,20]$ & 10212 & $F(0)$ & $15.81(7.36,33.96)$ \\
\hline 1.15.1 Treatment of pre-eclampsia/eclampsia & $2[16,20]$ & 10056 & $F(0)$ & $15.97(7.23,35.30)$ \\
\hline 1.15.2 Tocolysis & $1[18]$ & 156 & $\mathrm{~F}(\mathrm{NA})$ & $13.68(0.78,238.67)$ \\
\hline 1.16 Drowsiness or confusion & $3[19,20,26]$ & 11189 & $F(0)$ & $2.46(1.83,3.29)$ \\
\hline 1.16.1 Treatment of pre-eclampsia/eclampsia & $2[20,26]$ & 10127 & $F(0)$ & $2.26(1.06,4.85)$ \\
\hline 1.16.2 Fetal neuroprotection & $1[19]$ & 1062 & $F(N A)$ & $2.49(1.82,3.42)$ \\
\hline 1.17 Headache & $2[20,23]$ & 10556 & $F(0)$ & $2.21(1.27,3.86)$ \\
\hline 1.17.1 Treatment of pre-eclampsia/eclampsia & $1[20]$ & 9992 & $F(N A)$ & $2.12(1.19,3.76)$ \\
\hline 1.17.2 Fetal neuroprotection & $1[23]$ & 564 & $\mathrm{~F}(\mathrm{NA})$ & $3.89(0.44,34.57)$ \\
\hline 1.18 Thirst or mouth dryness & $2[19,20]$ & 11054 & $\mathrm{R}(42)$ & $2.38(1.59,3.56)$ \\
\hline 1.18.1 Treatment of pre-eclampsia/eclampsia & $1[20]$ & 9992 & $F(N A)$ & $3.36(1.72,6.58)$ \\
\hline 1.18.2 Fetal neuroprotection & $1[19]$ & 1062 & $F(N A)$ & $2.11(1.72,2.59)$ \\
\hline 1.19 Dizziness & $2[19,20]$ & 11054 & R (39) & $2.62(1.63,4.21)$ \\
\hline 1.19.1 Treatment of pre-eclampsia/eclampsia & $1[20]$ & 9992 & $\mathrm{~F}(\mathrm{NA})$ & $3.70(1.84,7.42)$ \\
\hline 1.19.2 Fetal neuroprotection & $1[19]$ & 1062 & $F(N A)$ & $2.21(1.53,3.19)$ \\
\hline \multicolumn{5}{|l|}{1.20 Sweating } \\
\hline 1.20.1 Fetal neuroprotection & $2[19,25]$ & 3265 & $\mathrm{R}(95)$ & $6.37(1.96,20.65)$ \\
\hline \multicolumn{5}{|l|}{1.21 Itching and/or tingling } \\
\hline 1.21.1 Treatment of pre-eclampsia/eclampsia & $1[20]$ & 9992 & $F(N A)$ & $14.98(1.98,113.38)$ \\
\hline \multicolumn{5}{|l|}{ 1.22 Blurred vision } \\
\hline 1.22.1 Fetal neuroprotection & $1[19]$ & 1062 & $F(N A)$ & $2.34(1.32,4.14)$ \\
\hline \multicolumn{5}{|l|}{1.23 Slurred speech } \\
\hline 1.23.1 Treatment of pre-eclampsia/eclampsia & $1[26]$ & 135 & $\mathrm{~F}(\mathrm{NA})$ & $3.04(0.13,73.42)$ \\
\hline
\end{tabular}


Table 1 Adverse effect estimates from randomised controlled trials (Comparison 1) (Continued)

\begin{tabular}{|c|c|c|c|c|}
\hline 1.24 Problems at the IV site or arm discomfort & $3[19,20,25]$ & 8704 & $\mathrm{R}(92)$ & $6.34(3.10,12.98)$ \\
\hline 1.24.1 Treatment of pre-eclampsia/eclampsia & $1[20]$ & 5439 & $F(N A)$ & $3.05(2.15,4.32)$ \\
\hline 1.24.2 Fetal neuroprotection & $2[19,25]$ & 3265 & $\mathrm{~F}(\mathrm{NA})$ & $9.11(7.18,11.55)$ \\
\hline \multicolumn{5}{|l|}{ 1.25 Problems at the IM site } \\
\hline 1.25.1 Treatment of pre-eclampsia/eclampsia & $1[20]$ & 4553 & $F(N A)$ & $1.49(1.25,1.79)$ \\
\hline 1.26 Caesarean section & $10[16-21,23-26]$ & 14105 & $F(0)$ & $1.04(1.00,1.08)$ \\
\hline 1.26.1 Treatment of pre-eclampsia/eclampsia & $6[16,17,20-22,26]$ & 10096 & $F(0)$ & $1.05(1.01,1.10)$ \\
\hline 1.26.2 Fetal neuroprotection & $3[19,23,25]$ & 3853 & $F(19)$ & $1.00(0.93,1.08)$ \\
\hline 1.26.3 Tocolysis & $1[18]$ & 156 & $\mathrm{~F}(\mathrm{NA})$ & $0.90(0.45,1.82)$ \\
\hline 1.27 Postpartum haemorrhage & $4[19,20,23,26]$ & 10535 & $F(0)$ & $0.94(0.87,1.04)$ \\
\hline 1.27.1 Treatment of pre-eclampsia/eclampsia & $2[20,26]$ & 8909 & $\mathrm{R}(43)$ & $1.31(0.39,4.41)$ \\
\hline 1.27.2 Fetal neuroprotection & $2[19,23]$ & 1626 & $F(0)$ & $0.84(0.61,1.15)$ \\
\hline 1.28 Pulmonary oedema & $4[20,21,24,25]$ & 12787 & $F(8)$ & $1.12(0.72,1.74)$ \\
\hline 1.28.1 Treatment of pre-eclampsia/eclampsia & $3[20,21,24]$ & 10560 & $F(0)$ & $0.95(0.60,1.57)$ \\
\hline 1.28.2 Fetal neuroprotection & $1[25]$ & 2227 & $F(N A)$ & $2.80(0.75,10.53)$ \\
\hline
\end{tabular}

$\mathrm{I}^{2}$ statistics is a test of heterogeneity; where $\mathrm{I}^{2}$ was $>30 \%$ summary estimates were calculated using random-effects meta-analysis; the bold effect estimates indicate statistical significance.

Abbreviations: $\mathrm{Cl}$ confidence interval, $F$ fixed-effect, $g$ gram, $I M$ intramuscular, $I V$ intravenous, $L D$ loading dose, $M D$ maintenance dose, NA not applicable

$R$ random-effects, $R R$ risk ratio.

\section{Adverse effects associated with treatment}

Women allocated to the lower dose regimen were significantly less likely to have absent tendon reflexes during treatment, an approximate $79 \%$ relative risk reduction (RR $0.21 ; 95 \%$ CI 0.10 to $0.46 ; 2$ trials, 176 women; Analysis 2.6). There were insufficient data for reliable conclusions about the differential effects on respiratory depression in one trial of 126 women (RR 0.25; 95\% CI 0.01 to 6.05; Analysis 2.5). There were no cases of gluteal abscess in one trial of 126 women measuring this outcome (Analysis 2.7).

\section{Other outcomes}

No significant differences were shown between groups in one trial of 126 women for the outcomes postpartum haemorrhage (RR 0.38 ; $95 \%$ CI 0.03 to 4.03; Analysis 2.8) and pulmonary oedema (RR 0.25 ; $95 \%$ CI 0.01 to 6.05 ; Analysis 2.9).

\section{Comparison 3: IV maintenance versus IM maintenance: prevention or treatment of eclampsia}

This comparison included three trials with 361 women (see Table 2 and Additional file 5 for effect estimates and forest plots for Comparison 3); all trials assessed magnesium sulphate for the prevention or treatment of eclampsia. Two trials compared Pritchard's IM regimen (a loading dose of $4 \mathrm{~g}$ IV and $10 \mathrm{~g}$ IM (5 g in each buttock), and $5 \mathrm{~g}$ IM in alternative buttocks every four hours as maintenance) with either a loading dose of $6 \mathrm{~g}$ IV, and $2 \mathrm{~g}$ /hour IV maintenance [30], or a loading dose of $4 \mathrm{~g}$ IV, and $0.75 \mathrm{~g} /$ hour IV maintenance [29]. The third trial did not describe its regimens, and compared the use of an IV Springfusor pump with standard hospital practice (IV loading dose; IM maintenance) [31].

\section{Life-threatening adverse effects of treatment}

In one trial, no significant difference was seen for the outcome maternal death (RR $0.35,95 \%$ CI 0.04 to 3.27; 1 trial, 137 women; Analysis 3.1). There were no data on the other primary outcomes.

\section{Interventions to limit adverse effects}

No clear difference was seen for the outcome discontinuation or modification of treatment due to adverse effects (RR 1.46, 95\% CI 0.83 to 2.58; 2 trials, 317 women; Analysis 3.2).

\section{Adverse effects associated with treatment}

No significant difference was seen in two trials for the outcome 'clinical signs of toxicity' (RR 0.82; 95\% CI 0.05 to $12.56 ; 154$ women; Analysis 3.3). In one trial of 300 women, women allocated to the IV regimen were almost five times more likely to report their pain level as 'acceptable,' compared with women allocated to the IM regimen (RR 4.93; 95\% CI 3.56 to 6.78; Analysis 3.4).

\section{Other outcomes}

There were insufficient data for reliable conclusions about the differential effects on caesarean section (RR 1.03; 95\% CI 0.78 to 1.35 ; 2 trials, 154 women; Analysis 3.5 ) and postpartum haemorrhage (RR $0.35 ; 95 \% \mathrm{CI} 0.04$ to 3.27; 1 trial, 137 women; Analysis 3.6). 
Table 2 Adverse effect estimates from randomised controlled trials (Comparisons 2-4)

\begin{tabular}{lllll}
\hline Outcome or subgroup & Studies & Participants & Method $\left(\mathrm{I}^{2}(\%)\right)^{*}$ & $\mathrm{RR}(95 \% \mathrm{Cl})$ \\
\hline Comparison $2:$ loc &
\end{tabular}

Comparison 2: lower dose versus higher dose magnesium sulphate IM maintenance: treatment of pre-eclampsia/eclampsia

2.1 Death due to 'toxicity'

2.1.1 4 g IV LD; 2 g/3 h IM MD versus Pritchard's regimen^

2.2 Stopped due to 'toxicity'

2.2.1 4 g IV LD; 2 g/3 h IM MD versus Pritchard's regimen^

2.3 Deferred or skipped doses

2.3.1 4 g IV LD; 2 g/3 h IM MD versus Pritchard's regimen^

2.3.2 'Dhaka' regimen* versus 'Bhalla' regimen

2.4 Given calcium gluconate

2.4.1 'Dhaka' regimen* versus 'Bhalla' regimen

2.5 Respiratory depression

2.5.1 4 g IV LD; 2 g/3 h IM MD versus Pritchard's regimen^

2.6 Absent tendon reflexes

2.6.1 $4 \mathrm{~g} \mathrm{IV} \mathrm{LD;} 2 \mathrm{~g} / 3 \mathrm{~h}$ IM MD versus Pritchard's regimen^

2.6.2 'Dhaka' regimen* versus 'Bhalla' regimen

2.7 Gluteal abscess (pain, phlebitis, inflammation)

2.7.1 4 g IV LD; 2 g/3 h IM MD versus Pritchard's regimen^

2.8 Postpartum haemorrhage

2.8.1 4 g IV LD; 2 g/3 h IM MD versus Pritchard's regimen^

2.9 Pulmonary oedema

2.9.1 4 g IV LD; 2 g/3 h IM MD versus Pritchard's regimen^

\begin{tabular}{|c|c|c|c|}
\hline $1[28]$ & 126 & $F(N A)$ & $0.25(0.01,6.05)$ \\
\hline $1[28]$ & 126 & $F(N A)$ & $0.05(0.01,0.39)$ \\
\hline $2[27,28]$ & 176 & $F(0)$ & $0.36(0.20,0.63)$ \\
\hline $1[28]$ & 126 & $F(N A)$ & $0.43(0.23,0.83)$ \\
\hline 1 [27] & 50 & $F(N A)$ & $0.23(0.07,0.71)$ \\
\hline 1 [27] & 50 & $F(N A)$ & $0.25(0.60,1.06)$ \\
\hline $1[28]$ & 126 & $F(N A)$ & $0.25(0.01,6.05)$ \\
\hline $2[27,28]$ & 176 & $F(0)$ & $0.21(0.10,0.46)$ \\
\hline $1[28]$ & 126 & $F(N A)$ & $0.20(0.08,0.50)$ \\
\hline 1 [27] & 50 & $F(N A)$ & $0.25(0.06,1.06)$ \\
\hline $1[28]$ & 126 & $F(N A)$ & No gluteal abscesses \\
\hline 1 [28] & 126 & $F(N A)$ & $0.38(0.03,4.03)$ \\
\hline $1[28]$ & 126 & $F(N A)$ & $0.25(0.01,6.05)$ \\
\hline
\end{tabular}

$1[28]-126 \quad F(N A)$

$0.25(0.01,6.05)$

Comparison 3: magnesium sulphate IV maintenance versus IM maintenance: treatment of pre-eclampsia/eclampsia

3.1 Death

\begin{tabular}{|c|c|c|c|c|}
\hline 3.1.1 4 g IV LD; 0.75 g/hour IV MD versus Pritchard's regimen^ & $1[29]$ & 137 & $F(N A)$ & $0.35(0.04,3.27)$ \\
\hline 3.2 Discontinuation or modification of treatment & $2[30,31]$ & 317 & $F(0)$ & $1.46(0.83,2.58)$ \\
\hline 3.2.1 $6 \mathrm{~g} \mathrm{IV} \mathrm{LD;} 2$ g/hour MD versus Pritchard's regimen^ & $1[30]$ & 17 & $F(N A)$ & $3.33(0.15,71.90)$ \\
\hline 3.2.2 'Springfusor pump' IV versus 'Standard' IM regimen & $1[31]$ & 300 & $F(N A)$ & $1.41(0.79,2.52)$ \\
\hline 3.3 Clinical signs of toxicity & $2[29,30]$ & 154 & R (38) & $0.82(0.05,12.56)$ \\
\hline 3.3.1 4 g IV LD; 0.75 g/hour IV MD versus Pritchard's regimen^ & $1[29]$ & 137 & $F(N A)$ & $0.21(0.01,4.27)$ \\
\hline 3.3.2 6 g IV LD; 2 g/hour MD versus Pritchard's regimen^ & $1[30]$ & 17 & $F(N A)$ & $3.33(0.15,71.90)$ \\
\hline \multicolumn{5}{|l|}{ 3.4 Pain level 'acceptable' } \\
\hline 3.4.1 'Springfusor pump' IV versus 'Standard' IM regimen & $1[31]$ & 300 & $F(N A)$ & $4.93(3.59,6.78)$ \\
\hline 3.5 Caesarean section & $2[29,30]$ & 154 & $F(0)$ & $1.03(0.78,1.35)$ \\
\hline 3.5.1 $4 \mathrm{~g}$ IV LD; 0.75 g/hour IV MD versus Pritchard's regimen^ & $1[29]$ & 137 & $F(N A)$ & $0.99(0.75,1.32)$ \\
\hline 3.5.2 $6 \mathrm{~g}$ IV LD; 2 g/hour MD versus Pritchard's regimen^ & $1[30]$ & 17 & $F(N A)$ & $1.50(0.47,4.76)$ \\
\hline \multicolumn{5}{|l|}{ 3.6 Postpartum haemorrhage } \\
\hline 3.6.1 4 g IV LD; 0.75 g/hour IV MD versus Pritchard's regimen^ & 1 [29] & 137 & $F(N A)$ & $0.35(0.04,3.27)$ \\
\hline
\end{tabular}

Comparison 4: short versus standard (24 hours) postpartum magnesium maintenance therapy: treatment of pre-eclampsia

$\begin{array}{lllll}\text { 4.1 Toxicity } & 2[32,33] & 256 & F(N A) & 0.25(0.06,1.08) \\ 4.1 .1 \text { Short (12 h) versus standard (24 h) } & 1[32] & 196 & F(N A) & \text { No toxicity } \\ \text { 4.1.2 Short (based on clinical criteria) versus standard (24 h) } & 1[33] & 60 & F(N A) & 0.25(0.06,1.08)\end{array}$


Table 2 Adverse effect estimates from randomised controlled trials (Comparisons 2-4) (Continued)

\begin{tabular}{|c|c|c|c|c|}
\hline \multicolumn{5}{|l|}{ 4.2 Side effects } \\
\hline 4.2.1 Short (based on clinical criteria) versus standard (24 h) & 1 [33] & 60 & $F(N A)$ & $0.17(0.02,1.30)$ \\
\hline \multicolumn{5}{|l|}{4.3 'Intolerance' } \\
\hline 4.3.1 Short (based on clinical criteria) versus standard (24 h) & 1 [33] & 196 & $F(N A)$ & No intolerance \\
\hline \multicolumn{5}{|c|}{ 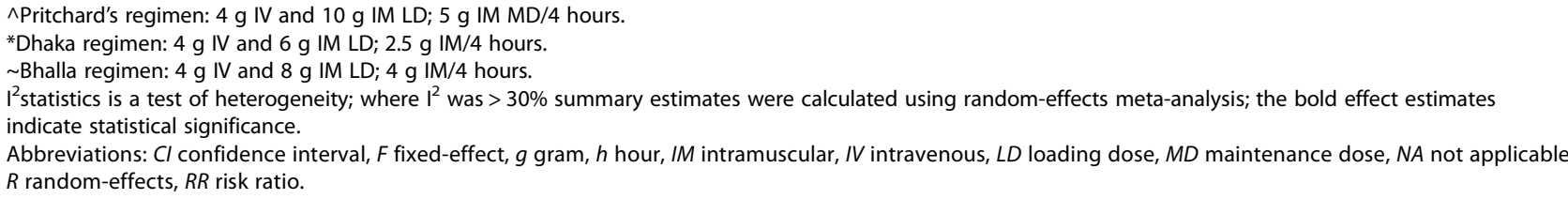 } \\
\hline
\end{tabular}

Comparison 4: short versus standard (24 hour) postpartum maintenance therapy: prevention of eclampsia

This comparison included two trials with 260 women which assessed magnesium sulphate for the prevention of eclampsia (see Table 2 and Additional file 5 for effect estimates and forest plots for Comparison 4). The trials compared individualised (short) versus standard (24 hour) postpartum maintenance therapy [32,33]. One trial compared $2 \mathrm{~g}$ /hour IV maintenance for 12 hours versus for 24 hours [32], whilst the other trial compared individualised maintenance (based on clinical criteria) with 24 hours maintenance; the regimens were unclear [33].

\section{Life-threatening adverse effects of treatment}

There were no data on the primary outcomes.

\section{Adverse effects associated with treatment}

There were insufficient data for reliable conclusions about the differential effects on 'toxicity' in two trials (RR 0.23; 95\% CI 0.06 to 1.08; 256 women; Analysis 4.1), or 'side effects' in one trial (RR $0.17 ; 95 \%$ CI 0.02 to 1.30; 60 women; Analysis 4.2). There were no cases of 'intolerance' among women in either group in the one trial of 196 women reporting this outcome (Analysis 4.3).

\section{Comparison 5: lower dose versus higher dose IV} maintenance: prevention of preterm birth

This comparison included two trials with 260 women (see Table 3 and Additional file 5 for effect estimates and forest plots for Comparison 5). Both trials assessed magnesium sulphate for the prevention of preterm labour, comparing a 4 g loading dose and 2 g/hour maintenance, with either a 6 g loading dose and $\geq 2$ g/hour maintenance [34] or a 4 g loading dose and 5 g/hour maintenance [35].

\section{Life-threatening adverse effects of treatment}

The two trials did not report on the review's primary outcomes.

\section{Interventions to limit adverse effects}

There was no cessation due to adverse effects in either trial (Analysis 5.1).

\section{Adverse effects associated with treatment}

No significant difference was shown for the outcome 'no side effects' when data for the two trials were pooled (RR 1.55; 95\% CI 0.94 to 2.84; 248 women; Analysis 5.2), however there was a substantial degree of statistical heterogeneity for this outcome $\left(\mathrm{I}^{2}=63 \%\right)$, and the subgroup interaction test indicated a potential differential effect based on the comparison regimen $\left(\mathrm{Chi}^{2}=2.73, \mathrm{P}=0.10\right.$, $\left.\mathrm{I}^{2}=63.4 \%\right)$. In one trial, women receiving the lower dose IV maintenance regimen ( 2 g/hour) were significantly more likely to experience 'no side effects' than women receiving the higher dose maintenance regimen ( $5 \mathrm{~g} /$ hour) (RR 1.96; 95\% CI 1.35 to 2.84; 148 women; Analysis 5.2.2). No significant difference was shown between the low and high dose IV maintenance groups for flushing (RR 0.61; 0.33 to 1.12 ; 2 trials, 248 women; Analysis 5.3), however moderate statistical heterogeneity was also observed for this outcome, which may be in part explained by the differing high dose comparison regimens.

There were insufficient data for reliable conclusions about the differential effects on the risk of nausea and vomiting (RR 0.79; 95\% CI 0.45 to $1.37 ; 1$ trial, 100 women; Analysis 5.4) and headache (RR 0.56; 95\% CI 0.30 to 1.05; 2 trials. 248 women; Analysis 5.5).

\section{Other outcomes}

No significant differences were shown between groups for the outcomes caesarean section (RR 1.11; 95\% CI 0.73 to 1.70 ; 2 trials, 248 women; Analysis 5.6) and pulmonary oedema (RR 0.21 ; $95 \%$ CI 0.03 to 1.76 ; 2 trials, 260 women; Analysis 5.7).

\section{Comparison 6: ready to use solution versus reference drug requiring dilution: prevention of preterm birth} This comparison included one trial of 46 women (see Table 3). The trial compared a pre-mixed 'ready-to-use' solution of magnesium sulphate, with a reference drug, a commercially available infusion solution concentrate requiring dilution [36]. All women were given a $4 \mathrm{~g}$ IV loading dose followed by 1-2 g/hour IV maintenance. 
Table 3 Adverse effect estimates from randomised controlled trials (Comparisons 5-6)

\begin{tabular}{|c|c|c|c|c|}
\hline Outcome or subgroup & Studies & Participants & Method $\left(I^{2}(\%)\right)^{*}$ & RR $(95 \% \mathrm{Cl})$ \\
\hline \multicolumn{5}{|c|}{ Comparison 5: lower dose versus higher dose magnesium sulphate IV maintenance: tocolysis } \\
\hline 5.1 Cessation due to adverse effects & $2[34,35]$ & 248 & $\mathrm{~F}(\mathrm{NA})$ & No cessation \\
\hline 5.2 No side effects & $2[34,35]$ & 248 & $R(63)$ & $1.55(0.94,2.58)$ \\
\hline 5.2.1 $4 \mathrm{~g} \mathrm{LD} ; 2 \mathrm{~g} / \mathrm{h}$ MD versus $6 \mathrm{~g} L D ; \geq 2 \mathrm{~g} / \mathrm{h} \mathrm{MD}$ & $1[34]$ & 100 & $F(N A)$ & $1.17(0.71,1.91)$ \\
\hline 5.2.2 $4 \mathrm{~g} \mathrm{LD} ; 2 \mathrm{~g} / \mathrm{h}$ MD versus $4 \mathrm{~g} L D ; 5 \mathrm{~g} / \mathrm{h}$ MD & $1[35]$ & 148 & $\mathrm{R}(\mathrm{NA})$ & $1.96(1.35,2.84)$ \\
\hline 5.3 Flushing & $2[34,35]$ & 248 & $\mathrm{R}(60)$ & $0.61(0.33,1.12)$ \\
\hline 5.3.1 $4 \mathrm{~g} \mathrm{LD} ; 2 \mathrm{~g} / \mathrm{h}$ MD versus $6 \mathrm{~g} L D ; \geq 2 \mathrm{~g} / \mathrm{h} \mathrm{MD}$ & $1[34]$ & 100 & $F(N A)$ & $0.87(0.46,1.63)$ \\
\hline $5.3 .24 \mathrm{~g} \mathrm{LD} ; 2 \mathrm{~g} / \mathrm{h}$ MD versus $4 \mathrm{~g}$ LD; $5 \mathrm{~g} / \mathrm{h}$ MD & $1[35]$ & 148 & $F(N A)$ & $0.46(0.29,0.73)$ \\
\hline \multicolumn{5}{|l|}{ 5.4 Nausea and vomiting } \\
\hline 5.4.1 $4 \mathrm{~g} \mathrm{LD} ; 2 \mathrm{~g} / \mathrm{h}$ MD versus $6 \mathrm{~g} L D ; \geq 2 \mathrm{~g} / \mathrm{h} \mathrm{MD}$ & $1[34]$ & 100 & $F(N A)$ & $0.79(0.45,1.37)$ \\
\hline 5.5 Headache & $2[34,35]$ & 248 & $F(0)$ & $0.56(0.30,1.05)$ \\
\hline 5.5.1 $4 \mathrm{~g} \mathrm{LD} ; 2 \mathrm{~g} / \mathrm{h}$ MD versus $6 \mathrm{~g} L D ; \geq 2 \mathrm{~g} / \mathrm{h}$ MD & $1[34]$ & 100 & $F(N A)$ & $0.80(0.23,2.81)$ \\
\hline 5.5.2 $4 \mathrm{~g} \mathrm{LD;} 2 \mathrm{~g} / \mathrm{h}$ MD versus $4 \mathrm{~g}$ LD; 5 g/h MD & $1[35]$ & 148 & $F(N A)$ & $0.50(0.24,1.03)$ \\
\hline 5.6 Caesarean & $2[34,35]$ & 248 & $F(0)$ & $1.11(0.73,1.70)$ \\
\hline 5.6.1 $4 \mathrm{~g} \mathrm{LD} ; 2 \mathrm{~g} / \mathrm{h}$ MD versus $6 \mathrm{~g} L D ; \geq 2 \mathrm{~g} / \mathrm{h}$ MD & $1[34]$ & 100 & $F(N A)$ & $1.31(0.78,2.21)$ \\
\hline $5.6 .24 \mathrm{~g} \mathrm{LD} ; 2 \mathrm{~g} / \mathrm{h}$ MD versus $4 \mathrm{~g}$ LD; 5 g/h MD & $1[35]$ & 148 & $F(N A)$ & $0.88(0.43,1.80)$ \\
\hline 5.7 Pulmonary oedema & $2[34,35]$ & 260 & $F(N A)$ & $0.21(0.03,1.76)$ \\
\hline 5.7.1 $4 \mathrm{~g} \mathrm{LD} ; 2 \mathrm{~g} / \mathrm{h}$ MD versus $6 \mathrm{~g} L D ; \geq 2 \mathrm{~g} / \mathrm{h} \mathrm{MD}$ & $1[34]$ & 100 & $F(N A)$ & No oedema \\
\hline 5.7.2 $4 \mathrm{~g} \mathrm{LD} ; 2 \mathrm{~g} / \mathrm{h}$ MD versus $4 \mathrm{~g} L D ; 5 \mathrm{~g} / \mathrm{h}$ MD & $1[35]$ & 160 & $F(N A)$ & $0.21(0.03,1.76)$ \\
\hline
\end{tabular}

Comparison 6: 'ready-to-use' magnesium sulphate solution versus a reference drug requiring dilution: tocolysis

6.1 Death

6.2 'Serious' adverse events

6.3 Withdrawn from the study due to adverse effects

6.4 Adverse events of 'severe intensity'

6.51 or 2 injection site changes

6.6 Poor general tolerability (physician assessed)

6.7 Respiratory depression

6.8 Warmth (mild, severe, very severe)

6.9 Nausea and/or vomiting (mild, severe, very severe)

6.10 Tiredness (mild, severe, very severe)

6.11 Headache (mild, severe, very severe)

6.12 Dry mouth (mild, severe, very severe)

6.13 Dizziness (mild, severe, very severe)

6.14 Sweating (mild, severe, very severe)

6.15 Skin redness (mild, severe, very severe)

6.16 Burning at injection site (mild, severe, very severe)

6.17 Palpitations (mild, severe, very severe)

6.18 Constipation (mild, severe, very severe)

6.19 Dyspnoea (mild, severe, very severe)

6.20 Heart pain (mild, severe, very severe)

6.21 Agitation (mild, severe, very severe)

\begin{tabular}{ll}
$1[36]$ & 46 \\
$1[36]$ & 46 \\
$1[36]$ & 46 \\
$1[36]$ & 4 \\
$1[36]$ & 46 \\
$1[36]$ & 4 \\
$1[36]$ & 46 \\
$1[36]$ & 4 \\
$1[36]$ & 4 \\
$1[36]$ & 4 \\
$1[36]$ & 4 \\
$1[36]$ & 4 \\
$1[36]$ & 4 \\
$1[36]$ & 4 \\
$1[36]$ & 4 \\
$1[36]$ & 4 \\
$1[36]$ & 4 \\
$1[36]$ & 4 \\
$1[36]$ & 4 \\
$1[36]$ & 4 \\
$1[36]$ & 4 \\
\hline 55 & 4
\end{tabular}

$F(N A)$
$F(N A)$
$F(N A)$
$F(N A)$
$F(N A)$
$F(N A)$
$F(N A)$
$F(N A)$
$F(N A)$
$F(N A)$
$F(N A)$
$F(N A)$
$F(N A)$
$F(N A)$
$F(N A)$
$F(N A)$
$F(N A)$
$F(N A)$
$F(N A)$
$F(N A)$
$F(N A)$

No deaths

No serious events

$0.67(0.12,3.62)$

$0.67(0.22,2.05)$

$1.00(0.28,3.52)$

$3.14(0.13,72.96)$

$0.20(0.10,3.95)$

$0.84(0.42,1.69)$

$0.536(0.11,2.56)$

$1.18(0.57,2.45)$

$0.92(0.41,2.06)$

$0.82(0.38,1.77)$

$1.05(0.30,3.64)$

$1.31(0.41,4.20)$

$1.75(0.48,6.38)$

$0.42(0.16,1.12)$

$1.58(0.29,8.46)$

$4.20(0.51,34.44)$

No dyspnoea

$0.35(0.20,8.10)$

$4.20(0.51,34.44)$

$1^{2}$ statistics is a test of heterogeneity; where $I^{2}$ was $>30 \%$ summary estimates were calculated using random-effects meta-analysis; the bold effect estimates indicate statistical significance.

Abbreviations: $C l$ confidence interval, $F$ fixed-effect, $g$ gram, $h$ hour, $I V$ intravenous, $L D$ loading dose, $M D$ maintenance dose, $N A$ not applicable, $R$ random-effects, $R R$ risk ratio. 
Figure 5 Risk of bias for non-randomised controlled trials. Risk of bias summary showing review authors' judgements about each risk of bias item for included non-randomised controlled trials. Each risk of bias item is judged as at a low risk of bias, unclear risk of bias or high risk of bias.

\section{Life-threatening adverse effects of treatment}

There were no maternal deaths, or 'serious' adverse events in either group (Analysis 6.1 and 6.2). There were no data on other primary outcomes.

\section{Interventions to limit adverse effects}

There were insufficient data for reliable conclusions about the differential effects on 'withdrawing from the study due to adverse effects' (Analysis 6.3) and one or two injection site changes (Analysis 6.5).

\section{Adverse effects associated with treatment}

There were insufficient data for reliable conclusions about the differential effects on any of the adverse effects reported in the trial (adverse events of severe intensity, poor general tolerability, respiratory depression, warmth, nausea and/or vomiting, tiredness, headache, dry mouth, dizziness, sweating, skin redness, burning at injection site, palpitations, constipation, dyspnoea, heart pain, agitation) (Analyses 6.4 and 6.6 to 6.21 ).

\section{Other outcomes}

The trial did not assess other outcomes of interest.

Evidence from non-randomised comparative studies with concurrent controls

Fourteen non-randomised comparative studies with concurrent controls were included (3,615 women) [37-51]: four non-randomised clinical trials (969 women) [46-49], four prospective before and after studies (78 women) [40-43], five retrospective cohort studies (2,502 women) [37-39,45,51] and one retrospective case-control study (66 women) [44]. Additionally, one historical control study was included (76 women) [50]. The detailed characteristics of the studies are presented in Additional file 4, with the risk of bias assessment presented in Figures 5 and 6 . For the four non-randomised trials, sequence generation, allocation concealment and blinding were not considered adequate (Figure 5). In regards to other comparative studies, for the majority of studies, selection, according to principles of the Newcastle-Ottawa Scale [14], was considered adequate (7/11), whilst comparability was largely unclear or not considered adequate, and outcome or exposure assessment was largely unclear (Figure 6).

Results from these studies largely supported those from the randomised trials (see Tables 4 and 5), with no major maternal complications (including death and cardiac arrest) in the studies that reported these outcomes. 


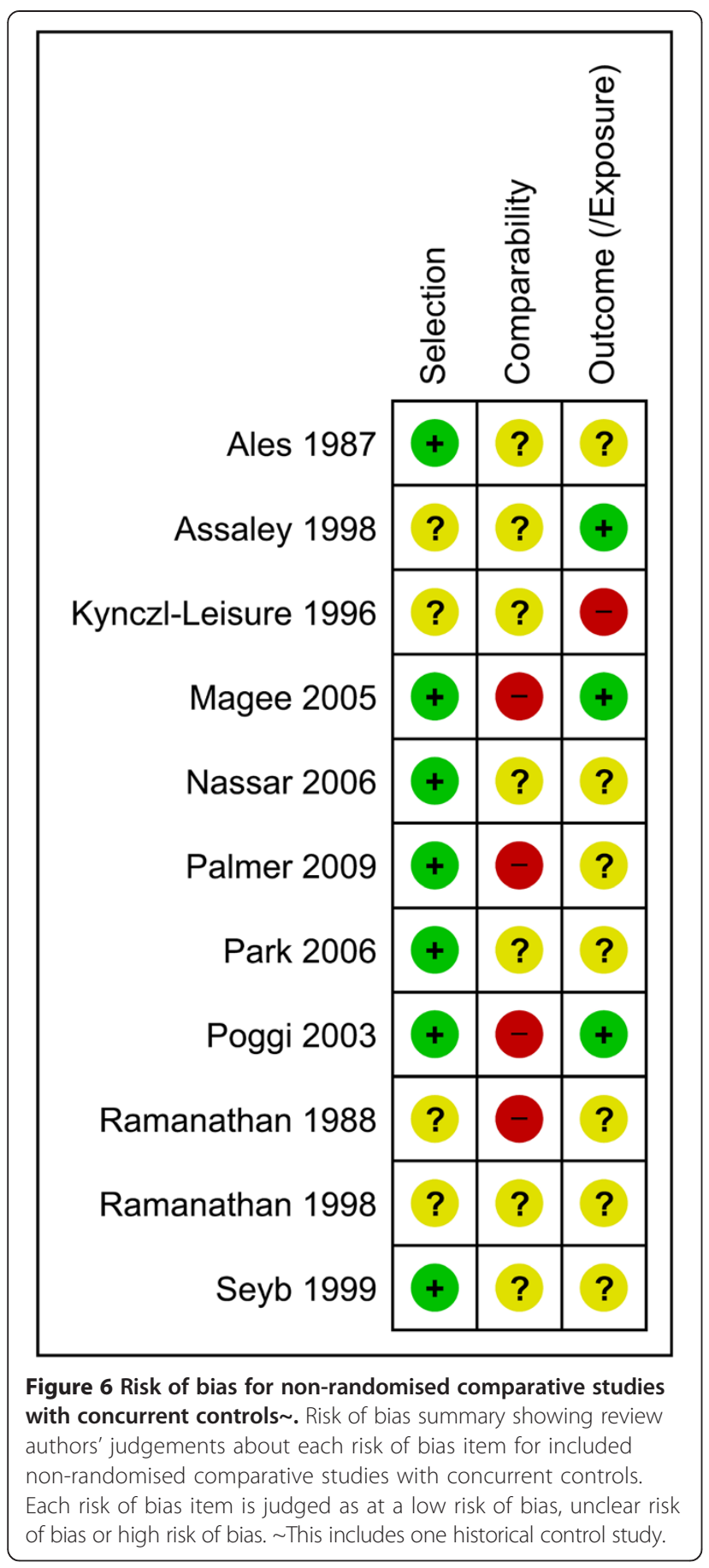

For one retrospective study reporting on the cessation of treatment due to adverse effects [45], the percentage of women stopping treatment was similar to the percentages reported in the randomised trials (Table 5). Effect estimates from non-randomised studies for the risk of caesarean section for women receiving magnesium sulphate versus no magnesium sulphate, were notably higher than the pooled effect estimate from the randomised trials (Table 4). In two retrospective cohort studies, women receiving magnesium sulphate were significantly more likely to experience failed labour induction [39], and undergo caesarean section due to failure to progress [37] (Table 4).

No significant increase in neuromuscular weakness among women receiving nifedipine as their antihypertensive during magnesium sulphate therapy, compared with women receiving an alternative or no antihypertensive agent, was shown in one retrospective cohort [45] (Table 4). Significantly increased risks of thirst, respiratory problems and minor bleeding, were however observed among women receiving nifedipine, compared with no antihypertensive agent; a significantly increased risk of neuromuscular blockade was observed among women receiving an alternative antihypertensive, compared with nifedipine [45] (Table 4). Whilst two prospective before and after studies showed a significant increase in bleeding time for women receiving magnesium sulphate $[40,41]$ (Table 4 ), this was not supported by an increased risk of postpartum haemorrhage in the randomised trials (Table 1). Similarly, whilst in one retrospective case control study, pregnant women with pulmonary oedema were significantly more likely to have received magnesium sulphate as compared with women without pulmonary oedema [44] (Table 4), the randomised trials did not support an increased risk of pulmonary oedema overall (Table 1).

In one retrospective study, women receiving magnesium sulphate for greater than 48 hours compared with for less than 48 hours had a significantly increased risk of experiencing more than one adverse effect [51] (Table 5). No significant differences were seen, however, in the risk of discontinuing therapy due to adverse effects, or for any other adverse effects [51] (Table 5). In one nonrandomised trial, women allocated to a loading dose only, compared with women receiving Pritchard's regimen (a loading dose of $4 \mathrm{~g}$ IV and $10 \mathrm{~g}$ IM, and $5 \mathrm{~g}$ IM every four hours as maintenance), were significantly less likely to experience nausea and vomiting, dizziness, irritation at the injection site, and undergo a caesarean section [49] (Table 5). Similar to the randomised trials, a significantly increased risk of pain was experienced among women receiving IM versus IV maintenance therapy [46] (Table 5). Supporting the findings from one randomised trial, no significant differences in adverse effects or medication errors were shown in the historical control study that assessed two different magnesium sulphate solutions using an identical dosage regimen [50] (Table 5).

\section{Evidence from case series}

Thirty-two studies [52-83] (3,276 women), 20 prospective and 12 retrospective in nature, reporting maternal adverse effects were included; the characteristics of the studies 
Table 4 Adverse effect estimates from comparative studies with concurrent controls

\begin{tabular}{|c|c|c|c|c|}
\hline Study & Participants and comparison & Adverse effect & Estimates & $\begin{array}{l}\text { P-value or effect estimate } \\
(95 \% \mathrm{Cl})\end{array}$ \\
\hline \multicolumn{5}{|c|}{ Magnesium sulphate versus no magnesium sulphate } \\
\hline \multirow[t]{2}{*}{ Ales 1987 [37] } & 178 women. $\mathrm{MgSO} 4$ for $\mathrm{H}(\mathrm{n}=64) \vee$ no MgSO4 $(\mathrm{n}=114)$ & Caesarean & $39.1 \vee 29.0 \%$ & AOR $2.81(1.99,3.62)^{\wedge}$ \\
\hline & & Caesarean (failure to progress) & $72.0 \% \vee 42.4 \%$ & OR $3.49(1.15,10.62)$ \\
\hline \multirow[t]{2}{*}{ Seyb 1999 [38] } & 1561 women. MgSO4 for PE $(n=54) \vee$ no MgSO4 $(n=1507)$ & Caesarean & $22.2 \% \vee 10.2 \%$ & OR $2.53(1.30,4.91)$ \\
\hline & & Caesarean & & AOR $2.18(1.04,4.55)^{*}$ \\
\hline Park 2006 [39] & 231 women. MgSO4 for PE $(n=29) \vee$ no MgSO4 $(n=202)$ & Failed induction of labour & & AOR $17.78(1.62,195.14) \sim$ \\
\hline Assaley 1998 [40] & 18 women. $\mathrm{MgSO} 4$ for $\mathrm{PE}(n=15) \vee$ no MgSO4 $(n=3)$ & $\begin{array}{l}\text { Significant } \uparrow \text { in bleeding time with } \mathrm{MgSO} 4 \\
\text { ( } v \text { no significant change with no MgSO4) }\end{array}$ & & $P<0.0043$ \\
\hline Kynczl-Leisure 1996 [41] & 12 women. $\mathrm{MgSO} 4$ for $\mathrm{PE}(n=9) \vee$ no MgSO4 $(n=3)$ & $\begin{array}{l}\text { Significant } \uparrow \text { in bleeding time with MgSO4 } \\
\text { ( } v \text { no significant change with no MgSO4) }\end{array}$ & & $P<0.01$ \\
\hline Ramanathan 1988 [42] & 16 women. $\mathrm{MgSO} 4$ for $\mathrm{PE}(n=10) \vee$ no MgSO4 $(n=6)$ & $\begin{array}{l}\text { Significant } \downarrow \text { in pulmonary function (FVC }(\mathrm{L}) \text {, } \\
\mathrm{FEV}_{1}(\mathrm{~L}) \text {, MW }(\mathrm{L}) \text { ) with } \mathrm{MgSO} 4 \text { ( } \vee \text { no significant } \\
\text { change with no MgSO4) }\end{array}$ & & $P<0.005 ; P<0.01 ; P<0.02$ \\
\hline Ramanathan 1988 [43] & $\begin{array}{l}32 \text { women. 1. Labour augmentation and MgSO4 for PE } \\
(\mathrm{n}=16) \vee 2 \text {. MgSO4 postpartum for PE }(n=6) \vee 3 \text {. Labour } \\
\text { induction and no MgSO4 }(n=10)\end{array}$ & $\begin{array}{l}\text { Depression of neuromuscular transmission for } \\
\text { Groups } 1 \text { and } 2 \text { (before } \mathrm{MgSO} 4 \text { to during } \mathrm{MgSO} \\
\text { v no changed for Group } 3 \text { (before and during } \\
\text { induction, and postpartum) }\end{array}$ & & \\
\hline Poggi 2003 [44] & $\begin{array}{l}66 \text { women. Pulmonary oedema }(n=15) \vee \text { no pulmonary } \\
\text { oedema }(n=51)\end{array}$ & $\begin{array}{l}\text { Case (pulmonary oedema) v control } \mathrm{MgSO} 4 \\
\text { exposure (for PE or PTL) }\end{array}$ & $93.3 \%$ v $62.7 \%$ & $P=0.049$ \\
\hline \multicolumn{5}{|c|}{ All women received magnesium sulphate: comparison based on antihypertensive agent received } \\
\hline \multirow[t]{13}{*}{ Magee 2005 [45] } & $\begin{array}{l}377 \text { women who all received MgSO4 for PE. 1. Nifedipine } \\
(n=162) \vee 2 \text {. Other antihypertensive }(n=32) \vee 3 \text {. No } \\
\text { antihypertensive }(n=183)\end{array}$ & Calcium gluconate given & $0.5 \% \vee 3.1 \% \vee 0.0 \%$ & $P=0.30(1 \mathrm{v} 2) ; P=0.47(1 \mathrm{v} 3)$ \\
\hline & & Infusion stopped due to adverse effects & $1.2 \% \vee 3.1 \% \vee 4.9 \%$ & $P=0.42(1 \mathrm{v} 2) ; P=0.05(1 \mathrm{v} 3)$ \\
\hline & & Infusion reduced due to adverse effects & $8.0 \% \vee 3.1 \% \vee 7.7 \%$ & $P=0.47(1 \vee 2) ; P=0.90(1 \vee 3)$ \\
\hline & & Neuromuscular weakness & $53.1 \% \vee 53.1 \% \vee 44.8 \%$ & $P=0.99(1 \mathrm{v} 2) ; P=0.13(1 \mathrm{v} 3)$ \\
\hline & & Absent deep tendon reflexes & $5.6 \% \vee 6.3 \% \vee 3.8 \%$ & $P=0.12(1 \mathrm{~V} 2) ; P=0.22(1 \mathrm{v} 3)$ \\
\hline & & Weakness & $15.4 \% \vee 28.1 \% \vee 10.9 \%$ & $P=0.99(1 \mathrm{~V} 2) ; P=0.26(1 \mathrm{v} 3)$ \\
\hline & & Respiratory depression & $9.9 \% \vee 9.4 \% \vee 6.6 \%$ & $P=0.99(1 \vee 2) ; P=0.45(1 \vee 3)$ \\
\hline & & Neuromuscular blockade & $0.0 \% \vee 6.25 \% \vee 0.0 \%$ & $P=0.03(1 \mathrm{v} 2) ; P=N A(1 v 3)$ \\
\hline & & Maternal hypotension & $41.4 \%$ v $31.3 \%$ v $53.0 \%$ & $P=0.33(1 \mathrm{v} 2) ; P=0.04(1 \mathrm{v} 3)$ \\
\hline & & Nausea/vomiting & $49.4 \%$ v $43.8 \%$ v $47.0 \%$ & $P=0.70(1 \mathrm{v} 2) ; P=0.66(1 \vee 3)$ \\
\hline & & Drowsiness/confusion & 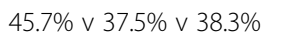 & $P=0.44(1 \vee 2) ; P=0.16(1 \vee 3)$ \\
\hline & & Dizziness & $28.4 \% \vee 25.0 \%$ v $20.8 \%$ & $P=0.83(1 \vee 2) ; P=0.10(1 \vee 3)$ \\
\hline & & Flushing & $22.2 \%$ v $15.6 \%$ v $20.8 \%$ & $P=0.48(1 \mathrm{v} 2) ; P=0.74(1 \mathrm{v} 3)$ \\
\hline
\end{tabular}


Table 4 Adverse effect estimates from comparative studies with concurrent controls (Continued)

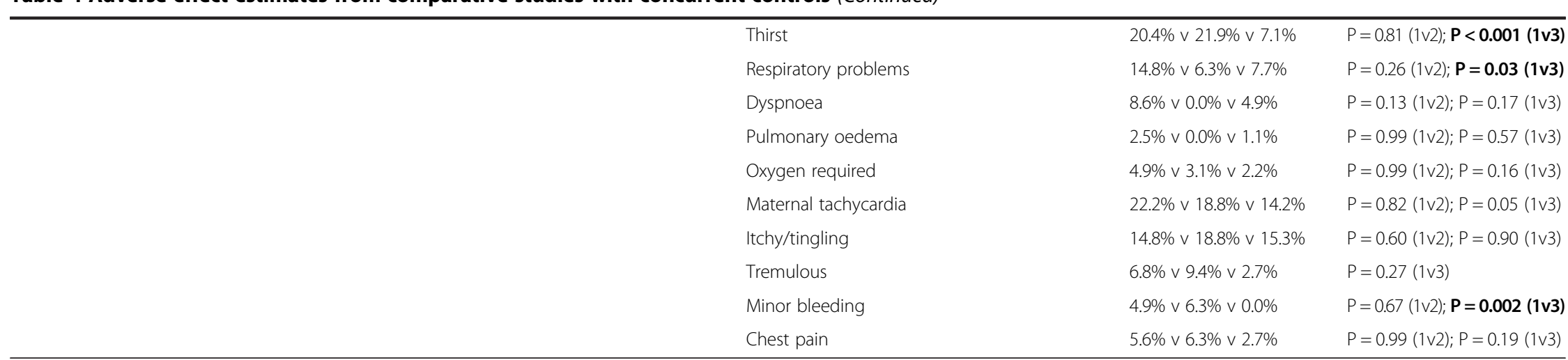

The bold effect estimates indicate statistical significance. ^ Logistic regression was used to adjust for age, race, parity, physician status, obesity, gestational age, and mean arterial pressure during labour; * “Controlling for the significant confounding variables"; "logistic regression analyses... adjusting for the potential confounding variables".

Abbreviations: $A O R$ adjusted odds ratio, $C l$ confidence interval, $F E V V_{1}$ forced expiratory volume at 1 second, $F V C$ forced vital capacity, $H$ hypertension, MgSO4 magnesium sulphate, MVV maximum voluntary ventilation NA not applicable, OR odds ratio, PE pre-eclampsia, PTL: preterm labour, v: versus, $\uparrow:$ increase, $\downarrow$ : decrease. 


\begin{tabular}{|c|c|c|c|c|}
\hline Study & Participants and comparison & Adverse effect & Estimates & Effect estimate $(95 \% \mathrm{Cl}$ \\
\hline \multicolumn{5}{|c|}{ Magnesium sulphate versus no magnesium sulphate } \\
\hline \multirow[t]{3}{*}{ Chowdhury 2000 [46] } & 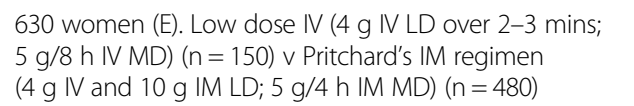 & Major adverse effects; respiratory depression & $0.0 \% \vee 0.0 \%$ & NA \\
\hline & & Absent knee jerks and oliguria; stopped dosing due to adverse effects & $0.0 \% \vee 3.2 \%$ & $\operatorname{RR} 0.10(0.01,1.71)$ \\
\hline & & Pain at injection site & $0.0 \% \vee 55.0 \%$ & RR $0.01(0.00,0.10)$ \\
\hline \multirow[t]{2}{*}{ Mahajan 2007 [47] } & $\begin{array}{l}95 \text { women (E). } 1 .(2 \mathrm{~g} I V \text { and } 4 \mathrm{~g} \mathrm{IM} \mathrm{LD;} 4 \mathrm{gIM} / 4 \mathrm{~h}) \\
(\mathrm{n}=37) \vee 2 .(2 \mathrm{~g} \text { IV and } 8 \mathrm{~g} \mathrm{IM} \mathrm{LD;} 4 \mathrm{~g} I \mathrm{IM} / 4 \mathrm{~h})(\mathrm{n}=58)\end{array}$ & Respiratory depression & $0.0 \% \vee 0.0 \%$ & NA \\
\hline & & Absent knee jerks and MD omitted & $56.8 \% \vee 31.0 \%$ & RR $1.83(1.14,2.94)$ \\
\hline \multirow[t]{3}{*}{ Young 1977 [48] } & $\begin{array}{l}144 \text { women (PE or E). } 1 . \text { ( } 10 \mathrm{~g} \text { IM LD; } 2 \text { g slow IV 'push' } \\
\text { with repeated doses every } 1-2 \mathrm{~h})(\mathrm{n}=97) \text { v } 2 \text {. } \\
(10 \mathrm{~g} \text { IM LD; continuous IV } 1 \mathrm{~g} / \mathrm{h})(\mathrm{n}=47)\end{array}$ & Death & $0.0 \% \vee 0.0 \%$ & NA \\
\hline & & Heat and flushing & $92.8 \% \vee 0.0 \%$ & RR $88.65(5.62,1397.80$ \\
\hline & & Respiratory effects (slowing respirations to complete apnoea) & $79.4 \% \vee 0.0 \%$ & RR $75.92(4.81,1198.55$ \\
\hline \multirow[t]{6}{*}{ Shoaib 2009 [49] } & $\begin{array}{l}100 \text { women (severe PE). LD only ( } 4 \mathrm{~g} \text { IV and } 10 \mathrm{~g} \text { IM LD) } \\
(\mathrm{n}=50) \vee \text { Pritchard's IM regimen ( } 4 \mathrm{~g} \text { IV and } 10 \mathrm{~g} \text { IM LD; } \\
5 \mathrm{~g} / 4 \mathrm{~h} \text { IM) }(\mathrm{n}=50)\end{array}$ & Death; respiratory failure or distress; cardiac arrest & $0.0 \% \vee 0.0 \%$ & NA \\
\hline & & Nausea and vomiting & $10.0 \% \vee 34.0 \%$ & RR $0.29(0.12,0.74)$ \\
\hline & & Warmth and flushing & $70.0 \% \vee 80.0 \%$ & $\operatorname{RR} 0.88(0.70,1.10)$ \\
\hline & & Dizziness & $20.0 \% \vee 56.0 \%$ & RR $0.36(0.19,0.65)$ \\
\hline & & Irritation at the injection site & $0.0 \% \vee 20.0 \%$ & RR $0.05(0.00,0.79)$ \\
\hline & & Caesarean & $12.0 \% \vee 30.0 \%$ & RR $0.40(0.17,0.95)$ \\
\hline \multirow[t]{4}{*}{ Palmer 2009 [50] } & $\begin{array}{l}76 \text { women (PE). New protocol ( } 20 \% \text { solution, separate } \\
\text { LD and MD bags) }(n=29) \vee \text { Old protocol } \\
(2-8 \% \text { solution, same } L D \text { and MD bag) }(n=47)\end{array}$ & Phlebitis; signs or symptoms of toxicity & $0.0 \% \vee 0.0 \%$ & NA \\
\hline & & Calcium gluconate (for hypocalcaemia) & $3.5 \% \vee 4.3 \%$ & RR $0.81(0.08,8.54)$ \\
\hline & & Errors (failure to reset pump after LD) & $0.0 \% \vee 4.3 \%$ & RR $0.32(0.02,6.44)$ \\
\hline & & Errors (change in drug order) & $3.5 \% \vee 2.1 \%$ & RR $1.62(0.11,24.92)$ \\
\hline \multirow[t]{5}{*}{ Nassar 2006 [51] } & $\begin{array}{l}155 \text { women (PTL). 1. Treatment for }>48 \text { hours } \\
(n=78) \vee 2 \text {. Treatment for }<48 \text { hours }(n=77)\end{array}$ & $\geq 1$ adverse effect & $30.8 \%$ v $15.6 \%$ & OR $2.41(1.10,5.26)$ \\
\hline & & Discontinuation due to adverse effects & $6.4 \% \vee 0.0 \%$ & OR $11.60(0.63,213.47)$ \\
\hline & & Chest tightness & $19.2 \% \vee 11.8 \%$ & OR $1.80(0.74,4.40)$ \\
\hline & & Visual disturbances & $6.4 \% \vee 1.3 \%$ & OR $5.21(0.59,45.63)$ \\
\hline & & Vulvar oedema & $1.3 \% \vee 0.0 \%$ & OR $3.00(0.12,74.79)$ \\
\hline
\end{tabular}


Table 5 Adverse effect estimates from comparative studies with concurrent controls (Continued)

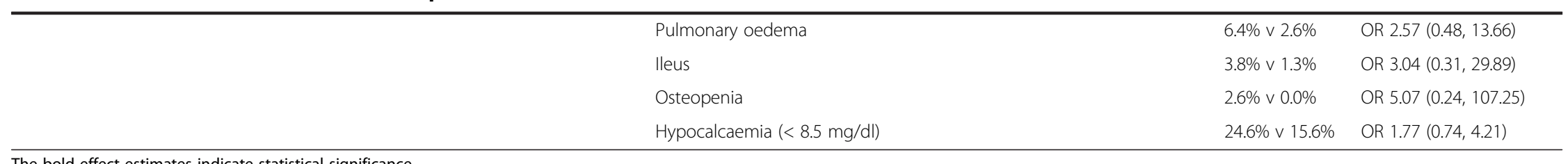

The bold effect estimates indicate statistical significance.

Abbreviations: $C l$ confidence interval, $E$ eclampsia, $g$ grams, $h$ hours, $I M$ intramuscular, $I V$ intravenous, $L D$ loading dose, MgSO4 magnesium sulphate, MD maintenance dose, mins minutes, OR odds ratio, $P E$ pre-eclampsia, PTL preterm labour, $R R$ risk ratio. 
and the quality assessment are presented in Additional file 4. Adverse effects have been presented in Table 6 as overall mean and median percentage estimates calculated from individual study results, with the range of percentages reported in the studies also presented. For the quality assessment of case series, we predominately considered participant selection, along with the collection/ reporting of adverse effect information, as detailed in Additional file 4.

Adverse effect estimates reported in case series were largely similar to those reported in the randomised trials in Comparison 1 (see Additional file 6). Mean/median percentage estimates of serious outcomes, respiratory arrest, the use of calcium gluconate and discontinuation of therapy due to adverse effects, were slightly higher in the case series. The estimates for the outcome 'any maternal adverse effects' reported in case series were however notably lower than those in the randomised trials. The case series estimates for nausea and/or vomiting, blurred vision and hypotension were higher than estimates from the randomised trials.

\section{Evidence from case reports}

Seventy-five studies describing a total of 137 case reports of maternal adverse effects were included [84-158] (see Table 7; the more detailed characteristics of cases are presented in Additional file 7).

Iatrogenic overdoses (16 reports) [84-100] and rapid administration of magnesium sulphate (one report) [86] were associated with a range of serious adverse events, such as respiratory arrest, cardiac arrest, cardiopulmonary arrest and death. Of note was a detailed account of 52 errors associated with magnesium sulphate administration; including seven cases resulting in death, or women remaining in a persistent vegetative state [87]. Additional errors, relating to the epidural or intrathecal administration of magnesium sulphate were associated with pain, inadequate pain relief, or temporary paralysis (four reports) [101-104].

Women at an increased risk of adverse effects were described (11 reports) (105-115), including women with renal failure (three reports) [113-115], and women with neuromuscular junction disorders, myopathies, and neuropathologies (eight reports) [105-112]. For the neurological cases, magnesium sulphate administration, according to recommended regimens, was associated with muscle pain, weakness or temporary paralysis, and associated respiratory problems. The interaction of magnesium sulphate with agents used in general anaesthesia (10 reports) [116-125] and with antihypertensive agents including nifedipine (six reports) [126-131] was associated with varying adverse effects, most commonly muscular weakness or paralysis, and altered respiratory function associated with prolonged neuromuscular blockade.
A variety of unusual maternal adverse effects, not previously attributed to magnesium sulphate, were described (11 reports) [132-142], including bilateral progressive labial swelling [132], marked osteoporotic change [136], and urinary tract stone formation [139]; in such cases women had received prolonged magnesium sulphate tocolysis therapy. Additional adverse effects, such as hypotension and fatigue as reported in randomised trials, were detailed (16 reports) [143-158]. Of note were five reports where adverse effects such as delirium [156], diplopia [153], tetany and paraesthesia [152-154] associated with serum hypocalcaemia, were described.

\section{Evidence from patient safety organisations}

Whilst the majority of patient safety organisations did not provide free access to their adverse event or equivalent databases, information regarding medication errors and maternal adverse effects associated with antenatal magnesium sulphate was available from the Institute for Safe Medication Practices USA. The Institute provided accessible information in their electronic medication safety newsletters. Three cases of serious iatrogenic overdose were reported in the 12 February 1997 issue [159]. Whilst the women survived, one suffered a respiratory arrest, and one, temporary paralysis of her extremities, ICU admission and the need for prolonged ventilation. Additional cases were described in the 30 June 1999, 15 June 2005, 20 October 2005, and 3 June 2010 issues.

The February 2010 issue of the Patient Safety Newsletter 'Sharing Lessons Leaned' produced by the Office of Safety and Quality in Health Care, Western Australia, reported 12 incidents involving magnesium sulphate overdose from October 2001 to October 2009, identified through searches of state-wide relevant databases [160]; the consequent maternal adverse effects were not however discussed.

\section{Women's experiences - from personal blogs and discussion forum threads}

(See Additional file 8 for blog and discussion forum thread sources).

\section{Before treatment}

Women considered information regarding others' experiences with magnesium sulphate important, with a particular desire for knowledge regarding adverse effects and their severity; a number of women expressed an explicit wish for "the truth, no matter how bad or scary." Women expressed expectations of treatment benefits, relating to the different indications for use, and also concern and a sense of fear regarding the possible adverse effects of treatment; one woman exclaimed "What?!! NO!!! ANYTHING BUT MAG!!!” 
Table 6 Adverse effect estimates from comparative studies with concurrent controls

\begin{tabular}{|c|c|c|c|c|c|}
\hline Adverse effect & Mean (\%) or effect & Median (\%) & Range (\%) & Women & Studies \\
\hline Death & 0.14 & 0.00 & 0 to 0.41 & 285 & Adewole 2000* [53]; Ekele 2005* [54]; Pritchard 1984* [55] \\
\hline Cardiac arrest & 0.00 & 0.00 & NA & 21 & Adewole $2000^{*}$ [53] \\
\hline Respiratory arrest & 0.41 & 0.42 & 0 to 0.82 & 983 & Adewole 2000* [53]; Pritchard 1984* [55]; Raman 1995* [56] \\
\hline Discontinuation due to adverse effects & 9.53 & 9.52 & $\begin{array}{l}1.75 \text { to } \\
20.78\end{array}$ & 532 & $\begin{array}{l}\text { Adewole 2000* [53]; Elliot 1983^ [57]; Girard 2005* [58]; } \\
\text { Harding 1997* [59]; Thapa 2008* [60] }\end{array}$ \\
\hline Given calcium gluconate & 0.70 & 0.70 & NA & 717 & Raman $1995^{*}[56]$ \\
\hline 'Toxicity' & 3.17 & 2.04 & 0.0 to 8.60 & 182 & $\begin{array}{l}\text { Dasari 2010* [61]; Donovan 1980* [62]; } \\
\text { Mojadidi 1969* [63]; Tukur 2010* [64] }\end{array}$ \\
\hline Need to adjust/skip dose due to adverse effects & 15.26 & 15.26 & $\begin{array}{l}5.26 \text { to } \\
25.26\end{array}$ & 114 & Ekele 2005* [54]; Getaneh 2010*^ [65] \\
\hline Respiratory depression & 1.67 & 0.72 & 0 to 4.76 & 1363 & $\begin{array}{l}\text { Adewole 2000* [53]; Ahmed 2004* [66]; Begum 2001* [67]; } \\
\text { Digre 1990^ [68]; Ekele 2005* [54]; Hales 1995^ [69]; } \\
\text { Harding 1997* [59]; Mojadidi 1969* [63]; Pritchard 1984* [55]; } \\
\text { Raman 1995* [56]; Sass 2007* [70] }\end{array}$ \\
\hline Absent or reduced deep tendon reflexes & 4.75 & 2.55 & 0 to 18.05 & 1789 & $\begin{array}{l}\text { Aali 2007* [52]; Begum 2001* [67]; Digre 1990^ [68]; Donovan 1980* [62]; } \\
\text { Ekele 2005* [54]; Hales 1995^ [69]; Omu 2008* [71]; Pritchard 1984* [55]; } \\
\text { Raman 1995* [56]; Sass 2007* [70] }\end{array}$ \\
\hline Any adverse effects & 13.39 & 14.29 & $\begin{array}{l}6.76 \text { to } \\
19.11\end{array}$ & 826 & Adewole 2000* [53]; Elliot 1983^ [57]; Omu 2008* [71] \\
\hline 'Minor side effects' & 1.75 & 1.75 & NA & 57 & Girard 2005* [58] \\
\hline Hypotension & 30.56 & 30.56 & NA & 72 & Hales 1995^ [69] \\
\hline Flushing or warmth & 52.88 & 52.88 & 4.55 to 100 & 27 & Cotton 1984* [72]; Harding 1997* [59] \\
\hline Nausea and/or vomiting & 47.37 & 38.46 & 3.66 to 100 & 373 & Cotton 1984* [72]; Digre 1990^ [68]; Elliot 1983^ [57] \\
\hline Generalised weakness & 23.08 & 23.08 & NA & 13 & Digre 1990^ [68] \\
\hline Drowsiness or confusion & 2.58 & 2.90 & 0.28 to 4.55 & 515 & Elliot 1983^ [57]; Harding 1997* [59]; Sass 2007* [70] \\
\hline Headache & 2.90 & 0.72 & 0.28 to 7.69 & 506 & Digre 1990^ [68]; Elliot 1983^ [57]; Sass 2007* [70] \\
\hline Blurred vision & 46.30 & 46.30 & $\begin{array}{l}0.28 \text { to } \\
92.31\end{array}$ & 368 & Digre 1990^ [68]; Elliot 1983^ [57] \\
\hline Diplopia & 30.77 & 30.77 & NA & 13 & Digre $1990 \wedge[68]$ \\
\hline Photophobia & 30.77 & 30.77 & NA & 13 & Digre $1990 \wedge[68]$ \\
\hline Visual signs & 76.92 & 76.92 & NA & 13 & Digre 1990^ [68] \\
\hline Abnormal visual acuity & 38.46 & 38.46 & NA & 13 & Digre $1990 \wedge[68]$ \\
\hline Impaired concentration-confusion & 23.08 & 23.08 & NA & 13 & Digre 1990^ [68] \\
\hline Cardiac arrhythmias & 23.08 & 23.08 & NA & 13 & Digre $1990 \wedge[68]$ \\
\hline Chest pain (and/or need for ECG) & 3.90 & 3.90 & 0.85 to 6.94 & 427 & Elliot 1983^ [57]; Hales 1995^ [69] \\
\hline Chest tightness & 0.28 & 0.28 & NA & 355 & Elliot 1983^ [57] \\
\hline
\end{tabular}


Table 6 Adverse effect estimates from comparative studies with concurrent controls (Continued)

\begin{tabular}{|c|c|c|c|c|c|}
\hline Delayed recovery from anaesthesia & 0.14 & 0.14 & NA & 717 & Raman $1995^{*}[56]$ \\
\hline Pulmonary oedema & 1.25 & 1.25 & 1.13 to 1.36 & 649 & Elliot 1983^ [57]; Yeast 1993*^ [73] \\
\hline Caesarean & 49.68 & 56.52 & $\begin{array}{l}32.53 \text { to } \\
60.00\end{array}$ & 225 & Aali 2007* [52]; Getaneh 2010*^ [65]; Pritchard 1984* [55] \\
\hline Caesarean due to labour induction & 48.91 & 51.85 & $\begin{array}{l}33.33 \text { to } \\
61.54\end{array}$ & 109 & Aali 2007* [52]; Getaneh 2010*^ [65]; Pritchard 1984* [55] \\
\hline 'Transient nausea, vomiting, headache, flushing and palpitations' & NA & NA & NA & 15 & Jirapinyo 1990^ [74] \\
\hline 'Magnesium toxicity suspected' & \multicolumn{4}{|c|}{$\begin{array}{l}\text { 2/49 deaths due to hypertensive disorders of pregnancy were } \\
\text { attributed to magnesium }\end{array}$} & Dasari $2010^{*}[61]$ \\
\hline \multirow[t]{2}{*}{ Hospital errors in obstetric patients } & \multicolumn{4}{|c|}{$\begin{array}{l}146 \text { hospital errors in obstetric patients ( } 3^{\text {rd }} \text { most common } \\
\text { obstetric drug resulting in patient harm) }\end{array}$} & Kfuri 2008 [75] \\
\hline & \multicolumn{4}{|c|}{$\begin{array}{l}10 \text { class } 2 \text { errors (need for additional treatment/ } \\
\text { hospitalisation) }\end{array}$} & Little $2001 \sim[76]$ \\
\hline 'Restrictive type of respiratory depression' & \multicolumn{3}{|c|}{ Sig $\downarrow$ in FVC $(L)$} & 18 & Bilgin 1994* [77] \\
\hline $\begin{array}{l}\text { Decrease in respiratory function - 'generalised respiratory } \\
\text { muscle weakness' }\end{array}$ & \multicolumn{3}{|c|}{ Sig $\downarrow$ MIP $(\mathrm{cm} \mathrm{H} 2 \mathrm{O})$, MEP $(\mathrm{cm} \mathrm{H} 2 \mathrm{O}), \mathrm{FEV}_{1}(\mathrm{~L})$} & 10 & Herpolsheimer 1991* [78] \\
\hline 'Reduced attention and rapid information processing ability' & \multicolumn{3}{|c|}{ Sig $\downarrow$ in SSS, PASAT, VAF, DSF scores } & 15 & Ghia 2000^ [79] \\
\hline \multirow{2}{*}{$\begin{array}{l}\text { Increase in bleeding time ('clinical significance remains to be } \\
\text { determined') }\end{array}$} & \multicolumn{3}{|l|}{ Sig $\uparrow$} & 104 & Fuentes 1995*^ [80], Guzin 2010* [81], Yazdani 2004^ [82] \\
\hline & \multicolumn{3}{|l|}{ NS $\uparrow$} & 40 & Moghadas 2007^ [83] \\
\hline
\end{tabular}

Values are presented as mean and median percentage estimates from case series, with the range of percentages reported.

*Women received MgSO4 for pre-eclampsia/eclampsia; ^ ^women received MgSO4 as a tocolytic agent; $\sim$ unknown indication for use.

Abbreviations: AE adverse effect(s), DSF Digits Span Forward (Wechsler Adult Intelligence Scale), FVC forced vital capacity, FEV, forced expiratory volume at 1 second, $L$ litres, MIP maximal inspiratory pressure,

MEP maximal expiratory pressure, NS not significant, PASAT Paced Auditory Serial Addition Tes, Sig significant, SSS Stanford Sleepiness Scale, VAF Verbal Associative Fluency Test, $\uparrow:$ increase, $\downarrow$ : reduction 
Table 7 Adverse effect from case reports

\begin{tabular}{|c|c|}
\hline Common theme and/or associated adverse effects & Studies \\
\hline \multicolumn{2}{|l|}{ latrogenic overdose (16 studies) } \\
\hline Death & Anon 1990 [84]; Cohen 1992 [85]; Richards 1985 [86] \\
\hline Death or persistent vegetative state & Simpson 2004 (7 cases) [87] \\
\hline Cardiopulmonary arrest & $\begin{array}{l}\text { McCubbin } 1981 \text { [88]; McDonnell } 2009 \text { [89]; Morisaki } 2000 \text { [90]; } \\
\text { Rabinerson } 1994 \text { [91]; Swartjes } 1992 \text { [92] }\end{array}$ \\
\hline Cardiac arrest & Cohen 1992 [85] \\
\hline Respiratory arrest & Bohman 1990 [93]; Cao 1999 [94]; McKenna 2006 [95]; Wax 1995 [96] \\
\hline "Life-threatening situation" & Bruhwiler 1994 [97] \\
\hline Coma & Hayashi 2003 [98] \\
\hline Ventilatory impairment; failure to rouse from general anaesthesia & McDonnell 2010 [99] \\
\hline Need for additional monitoring & Buettner 2010 (2 cases) [100] \\
\hline Variety (not death or remaining in a persistent vegetative state) & Simpson 2004 (45 cases) [87] \\
\hline \multicolumn{2}{|l|}{ Rapid administration (1 study) } \\
\hline Cardiac arrest & Richards 1985 [86] \\
\hline \multicolumn{2}{|l|}{ Unintended epidural or intrathecal administration (4 studies) } \\
\hline Bilateral periumbilical pain & Dror $1987[101]$ \\
\hline Inadequate pain relief & Goodman 2006 (2 cases) [102] \\
\hline Paralysis of lower extremities & Lejuste 1985 [103]; Lewis-Younger 2004 [104] \\
\hline
\end{tabular}

Increased risk of adverse effects - neuromuscular junction disorders, myopathies and neuropathologies (8 studies)

Weakness and/or temporary paralysis

Muscle pain and damage

Acute respiratory insufficiently; ventilatory failure; respiratory depression

"Magnesium toxicity"

Increased risk of adverse effects - renal failure (3 studies)

Decreased or absent deep tendon reflexes; prolonged QT interval

Muscle weakness

Progressive quadriparesis

\section{Drug interactions - agents used in general anaesthesia (10 studies)}

Cardiac arrest

Respiratory arrest

Failure to achieve adequate ventilation

Numb; difficultly moving upper extremities

Prolonged neuromuscular blockade

\section{Drug interactions - other agents (6 studies)}

Neuromuscular blockade - muscle weakness or paralysis

Extreme bradycardia

Severe hypotension

\section{Unusual/unexpected adverse effects (11 studies)}

Bilateral, progressive labial swelling (need for caesarean)

Worsened clinical picture of appendicitis and cholecystitis

Impaired lactogenesis

Severe paralytic ileus

Marked osteoporotic change (hips, knees, ankles)

Breast engorgement and galactorrhea
Bashuk 1990 [105]; Bruner 1990 [106]; Catanzarite 2008 [107]

Hosono 2001 [108]

Cohen 1976 [109]; Mueksch 2007 [110]; Robins 2007 [111]

Moriarty 2008 [112]

Archer 2010 [113]

Chan 2008 [114]

Nethravathi 2007 [115]

Saitoh 1994 [116]

Baraka 1984 [117]

Nguyen 2001 [118]

Fay 1996 [119]

Funai 2010 [120]; Hino 1997 [121]; Kwan 1996 [122]; Sinatra 1985 [123]; Yoshida 2006 (2 cases) [124]; Sloan 2001 [125]

Ben-Ami 1994 [126]; Snyder 1989 [127]; Wu 2010 [128]

Pittman 2000 [129]

Scardo 1997 [130]; Waisman 1998 (2 cases) [131]

Awwad 1994 [132]

Basaran 2007 [133]

Haldeman 1993 [134]

Hill 1985 [135]

Hung 2005 [136]

Lurie 2002 [137] 
Table 7 Adverse effect from case reports (Continued)

\begin{tabular}{ll}
\hline Development of central pontine myelinolysis & Riggs 2000 [138] \\
Urinary tract stone (magnesium ammonium phosphate) & Sameshima 1997 [139] \\
Hyperkalaemia and hyponatremia (hyporeninemic hypoaldosteronism) & Spital 1991 [140] \\
Left retinal detachment; partial right detachment & Roberts 1998 [141] \\
Extensive urticarial rash & Thorp 1989 (2 cases) [142] \\
Other adverse effects (16 studies) & \\
Severe hypotension & Bourgeois 1986 (2 cases) [143]; Rodis 1987 [144] \\
Hypothermia & Cardosi 1998 [145]; Rodis 1987 [144] \\
Bradycardia (39-44/minute) & Hennessy 1999 [146] \\
Asymptomatic atrial fibrillation (100-150/minute) & Oettinger 1993 [147] \\
Absent deep tendon reflexes & Pritchard 1979 [148] \\
Marked weakness; difficulty breathing & Pritchard 1979 [148] \\
Sleepiness/fatigue; depressed/absent deep tendon reflexes & Herschel 2001 [149]; Tang 2010 [150] \\
Chest pain; inverted T waves (ECG) (transient subendocardial ischemia) & Sherer 1992 [151] \\
Pulmonary oedema & Elliot 1979 [157]; Worrell 1992 [158] \\
Bilateral hand contractures; tetany (serum hypocalcaemia) & Koontz 2004 (2 cases) [152] \\
Diplopia; malaise; paresthesia; hoarseness; tetany (serum hypocalcaemia) & Mayan 1999 (2 cases) [153] \\
Hypotension; cyanosis; tetany (serum hypocalcaemia) & Monif 1972 [154] \\
Chest tightness and pain; prolonged QT interval (serum hypocalcaemia) & Nassar 2007 [155] \\
Delirium with myoclonus (serum hypocalcaemia) & Ganzenvoort 2002 [156] \\
\hline
\end{tabular}

\section{During treatment}

Whilst variations in experiences existed, women described a variety of adverse effects, the predominant being a sensation of heat: "MY GOD, THE HEAT! I felt so hot and it came from within." Other commonly described adverse effects were similar to those frequently reported adverse effects in randomised trials - muscle weakness, lethargy, blurred vision, nausea or vomiting, headaches, confusion, arm discomfort, thirst, feeling sweaty and dizzy. Less common adverse effects women attributed to magnesium sulphate treatment included a failed induction of labour and consequent need for a caesarean section, the delayed onset of lactation, and the development of pulmonary oedema. A number of women described their request or the need for treatment cessation due to adverse effects, with two women highlighting their experience of being "accidentally overdosed." Many women portrayed their experience as terrifying, revealing a sense of anguish.

\section{After treatment}

Women expressed relief following treatment cessation, and great concerns regarding the possibility of treatment in future pregnancies: "I pray I never have to do it again." A number of women felt information regarding the possible adverse effects of treatment was miscommunicated and misleading. Although many women recalled adverse effects of severe intensity, they were generally very thankful, and there was agreement among commenting women that the potential perceived benefits of treatment outweighed discomforts experienced, with magnesium sulphate being described as "a necessary evil."

\section{Discussion}

It is widely acknowledged that the use of antenatal magnesium sulphate, for the prevention of eclampsia in women with pre-eclampsia and the treatment of women with eclampsia [1-4,9], to delay or prevent preterm birth $[5,6]$, and for neuroprotection of the fetus when given to women at risk of preterm birth [7], may be associated with adverse effects for the mother. The risk of individual adverse effects, and how such adverse effects vary according to different regimens, has however not been clear. Determining this is of great importance given the significant number of women who may be eligible for treatment with magnesium sulphate during pregnancy with approximately 2-8\% of pregnancies complicated by pre-eclampsia [161], and an estimated $1-2 \%$ of births being very preterm (before 32 weeks' gestation) [162].

\section{Summary of main results}

We were able to include 21 randomised trials in this systematic review; 11 comparing magnesium sulphate with placebo or no treatment, and 10 comparing different magnesium sulphate regimens. Whilst 'good' data on well recognised and easily detectable adverse effects may 
be potentially available from randomised trials, the often small numbers of participants, who may differ from individuals given the treatment in everyday practice, or the short periods of time that the participants are studied for, can reduce the possibility of unpredictable, rare or delayed adverse effects being observed and reported $[12,163,164]$. For such reasons, it was considered important to include other study designs. We included 15 non-randomised comparative studies with concurrent controls, 32 case series, and 75 papers describing individual case reports.

Evidence from the randomised trials assessing magnesium sulphate versus placebo or no treatment confirmed the expected higher rates of 'minor' maternal adverse effects among women receiving treatment, without an increase in major complications (death, cardiac arrest, respiratory arrest). The four randomised trials reporting on 'any adverse effects' of treatment, showed an absolute risk of $38 \%(2,521 / 6,642)$ for women exposed to antenatal magnesium sulphate compared with $8.5 \%(567 / 6,680)$ for women unexposed. Five randomised trials reported on the need for cessation of treatment due to maternal adverse effects, with significantly more women receiving magnesium sulphate having their therapy stopped compared with women not receiving magnesium sulphate $(6.6 \%$ versus $2.4 \%$ ). The most frequently reported adverse effects included warmth or flushing, sweating, and arm discomfort or problems at the IV site.

When considering indication for use and regimens for administration, very few differences were observed between the pre-specified subgroups. Considering indication for use, no significant subgroup interactions were identified, expect for when considering arm discomfort, which suggested a possible increase for women receiving treatment for fetal neuroprotection (compared with women receiving treatment for pre-eclampsia). When considering regimen for administration for the trials comparing magnesium sulphate with placebo or no treatment, the subgroup interaction tests did not indicate differential effects by treatment subgroups. For the majority of outcomes, the subgroups contained very few trials (many contained only one), making comparisons between subgroups difficult.

The 10 randomised trials comparing different magnesium sulphate regimens were mostly too small to provide reliable evidence about the comparative effects of different regimens on maternal adverse effects. Whilst this review did not formally assess efficacy, none of the trials demonstrated significantly decreased effectiveness with the lower dose regimens. One trial reporting on the cessation of treatment due to adverse effects showed significantly more women receiving higher dose IM maintenance having their therapy ceased as compared with women receiving lower dose IM maintenance $(25.9 \%$ versus $1.4 \%)$. This was supported by one small non-randomised trial, reporting significantly higher rates of adverse effects for women receiving Pritchard's IM regimen versus a loading dose only. One randomised trial showed significantly more women receiving magnesium sulphate via an IV pump reporting 'acceptable' pain as compared with women receiving magnesium sulphate via the IM route $(100 \%$ versus $20 \%$ ). This was supported by a non-randomised trial that showed a significantly higher risk of pain among women receiving magnesium sulphate via the IM route versus via an IV infusion.

The data from the 32 case series largely supported that from the randomised trials. Whilst serious outcome estimates were broadly similar, differences between procedures for collecting information on adverse events between studies may help to explain the variations observed between estimates. Higher estimates for 'any adverse effects' in randomised trials may have been influenced by the use of more rigorous methods for data collection, such as the use of trial-specific check-lists [19]. The notably higher estimates in case series for blurred vision and nausea and/or vomiting similarly may have been influenced by the use of specific questioning and interviewing in regards to adverse effects [68], and incomplete reporting, such as the use of generic statements [72].

As the establishment of a causal relationship between a treatment and a subsequent adverse effect through individual cases is difficult, data presented in case reports should be interpreted with a degree of caution. That said, the significant harm that may result from accidental overdose of magnesium sulphate is unquestionable. The 16 reports, including Simpson's account of 52 errors associated with administration [87], highlight the potentially lifethreatening consequences of magnesium sulphate overdose.

Factors contributing to iatrogenic overdoses were identified [87]. These included: the inaccurate or inadequate mixing of an IV magnesium sulphate solution, leading to a higher concentration of solution than intended or an undesirable concentration gradient between the infusion bag and tubing; the use of infusion bags containing a high total dose of magnesium sulphate, and the consequent danger associated with infusion rate programming error; the use of the same infusion bag for administration of the loading and maintenance infusions, and the failure to reduce the infusion rate following the loading dose; the removal of an IV line from an IV pump temporarily, and the accidental free-flow of solution; the handover of clinical responsibilities in busy units, and the transfer of women between units. Fortunately in most reported cases, the error was recognised before permanent adverse outcomes occurred. Monitoring of maternal status (before, during and after administration of magnesium sulphate), to allow signs and symptoms of toxicity to be recognised, and for potential consequences of errors to be mitigated in a timely manner, is imperative. Common 
recommendations for monitoring include: regular assessment and documentation of vital signs (pulse, blood pressure, respiratory rate), level of consciousness, patellar reflexes and urinary output; and the provision of 1:1 nursing care during loading administration (and 1:2-3 care during maintenance or where $1: 1$ care is not possible) [87].

Case reports also highlighted the potential for an increased risk of adverse effect occurrence among particular groups of women - those with renal insufficiency or failure, and those with neuromuscular disorders such as myasthenia gravis, Friedreich's ataxia, myotonic dystrophy, and other rare myopathies, such as mitochondrial myopathy. The potential for prolonged/enhanced neuromuscular blockade when magnesium sulphate is used in conjunction with general anaesthetic agents and antihypertensive agents including nifedipine was also shown. Whilst this potential interaction between magnesium sulphate and nifedipine was highlighted, reassuringly no increased neuromuscular blockade was shown in the retrospective cohort study that compared the use of nifedipine versus other/no antihypertensive during magnesium sulphate treatment (Table 4). Though an excess of respiratory problems and minor bleeding was shown among women receiving nifedipine, compared with no antihypertensive, the condition of women receiving nifedipine was considered comparatively more severe (more severe hypertension, more frequent pre-eclampsia symptoms), which was suggested to account for this difference [45].

Adverse effects not previously reported in randomised trials that were discussed in case reports included marked bilateral labial swelling, severe paralytic ileus, marked osteoporotic change, and urinary tract stone formation $[132,135,136,139]$. In each case, magnesium sulphate had been used as a tocolytic agent for a prolonged period (nine, three, 101 and 21 days respectively). The relevant Cochrane reviews have shown magnesium sulphate to be ineffective at delaying or preventing preterm birth $[5,6]$. Acknowledging this and the maternal adverse effects of magnesium sulphate, from minor to unpleasant to potentially fatal, its use as a tocolytic appears inappropriate, as has been strongly stated by Grimes and Nanda [165]. Furthermore, the Food and Drug Administration in the United States recently advised health care professionals against the use of magnesium sulphate as a tocolytic for more than five to seven days due to associated harm to developing fetal bones (the use of the drug for tocolysis is 'off-label' (not approved by the Food and Drug Administration)) [166]. In view of the extremely widespread use of antenatal magnesium sulphate in obstetric practice, the potential neonatal and infant adverse effects of antenatally administered magnesium sulphate additionally require comprehensive evaluation, such as by systematic review.

\section{Overall completeness, applicability and quality of the evidence}

This review was based on a comprehensive search strategy, and whilst no language restrictions were applied, inclusion was restricted to those studies written in English or for which a translation was readily available, and to those studies published in the databases that were searched, which may have limited available studies. Additionally, there were a number of articles that were excluded as we were unable to obtain the abstract and/or full-text. The included studies were however conducted in both high-income and low-income countries, and are therefore may considered widely applicable to the treatment of pregnant women.

The main limitations include the small number of studies with relatively small sample sizes comparing different antenatal magnesium sulphate regimens, and the missing data for several important outcomes in almost all trials. Additionally, a great number of trials could not be included in this review, as they did not provide any information regarding maternal adverse effects of treatment. This supports previous reports, that many trials do not report harms, or do so in a fragmented or suboptimal way [167].

The studies included in the review were of mixed quality and we emphasise the need to consider the risk of bias as outlined in Figures 2, 3, 4, 5 and 6, and the more detailed quality assessment in Additional file 4, which includes consideration of the different procedures used to collect information on adverse effects and reporting, when interpreting results.

\section{Potential biases in the review process}

We recognise the potential for bias associated with the second reviewer reviewing a random sample of the search records $(10 \%$ of the total), and independently extracting data from a random sample of included studies (10\% of the total, and all 21 randomised controlled trials). We attempted to minimise bias however in a number of ways, for example by using a comprehensive search strategy. Although this was extensive, it is possible that some studies conducted in low- and middle-income countries may not have been identified, if they were not published, or published in journals not indexed in the bibliographic databases searched.

\section{Agreements and disagreements with other reviews}

We are not aware of any other reviews specifically assessing maternal adverse effects of antenatal magnesium sulphate, and comparing the adverse effects of different regimens for the various indications for use. The findings of this review are broadly consistent with results from relevant Cochrane reviews, comparing magnesium sulphate with alternative drugs for women with eclampsia 
[1-3], evaluating magnesium sulphate for women with preeclampsia [4], assessing magnesium sulphate for preventing preterm birth in and after threatened preterm labour [5,6], and evaluating magnesium sulphate for neuroprotection of the fetus for women at risk of preterm birth [7].

These Cochrane reviews similarly concluded no increased risk of major maternal complications (death, cardiac arrest, respiratory arrest) for women receiving magnesium sulphate according to those regimens used in the trials $[4,5,7]$, increased risks of many comparatively minor adverse effects [4,6,7], and an increased risk of women ceasing treatment due to adverse effects [7]. One Cochrane review that assessed magnesium sulphate for women with pre-eclampsia had previously shown a small increased risk of caesarean section for women receiving magnesium sulphate, compared with placebo/no treatment [4], as was suggested in this review.

The findings of this review largely support the methodological conclusions from the systematic review by Golder et al. [10] - that there are often limited differences in the risk estimates of adverse effects derived from randomised trials and observational studies. Further, they highlight the importance of including a broad range of study designs to ensure that the most complete picture of potential harms associated with an intervention can be drawn [10,163,167].

\section{Conclusions}

\section{Implications for practice}

By considering adverse effects alone, this review was not designed to guide the choice of magnesium sulphate regimen for the different antenatal indications for use. Healthcare providers must therefore weigh the potential risks of magnesium sulphate for the mother against the benefits, for each known beneficial indication separately.

Vigilance in the use of magnesium sulphate is required to promote and ensure safety for women. Certainly, errors associated with the administration of magnesium sulphate represent a significant and perhaps unappreciated risk of harm. An important step in improving the safety for women is knowledge and recognition of the risk of error; this review, along with articles such as that published by Simpson [87], may help in increasing awareness. Individual hospitals should consider common precursors, or contributing factors, to errors associated with antenatal magnesium sulphate occurring in their obstetric units, and ensure safety procedures are in place to help prevent such accidents.

It is important, where ever possible, that women receive a full explanation, not only of why antenatal magnesium sulphate is in their case needed, but also of the nature of symptoms that may be experienced during treatment. An understanding of these benefits, the possible adverse effects, and the vigilant care and monitoring that will be received during treatment, may help to decrease or relieve anxiety during treatment that may otherwise be caused by unexpected adverse effects. Health professionals should seek to make their approach to information provision open and honest.

\section{Implications for research}

Fewer maternal adverse effects may be a benefit of lower dose magnesium sulphate regimens, and whilst this review did not formally assess effectiveness, no trial included in this review showed compromised efficacy with such lower dose regimens. Further trials comparing different regimens are however required, to determine the optimal regimens that achieve the desired clinical effectiveness with minimal maternal adverse effects and cessation, for each of the known beneficial indications for use. Such trials must be of a high quality, and of sufficient sample size to assess the comparative effects on relevant maternal and/or infant efficacy and safety related outcomes. For all future randomised trials, consideration of the extension for harms of the CONSORT statement [15] including during the study design phase, is crucial to ensure the better collection and reporting of adverse effects [167].

A number of important questions remain regarding maternal adverse effects of magnesium sulphate. Common reasons for ceasing treatment, and how serious adverse effects and treatment cessation vary according to total dose of magnesium sulphate received, require evaluation. Such questions may be addressed in an individual patient data meta-analysis. Additional well-designed and sufficiently powered trials or observational studies are required to address the need to reduce errors associated with magnesium sulphate administration. Such studies may, for example, consider comparing the use of a pre-mixed solution with a concentrate requiring dilution, or the use of separate infusion bags for the loading and maintenance dose infusions, compared with the use of the same infusion bag with the need to reprogram infusion rates.

\section{Additional files}

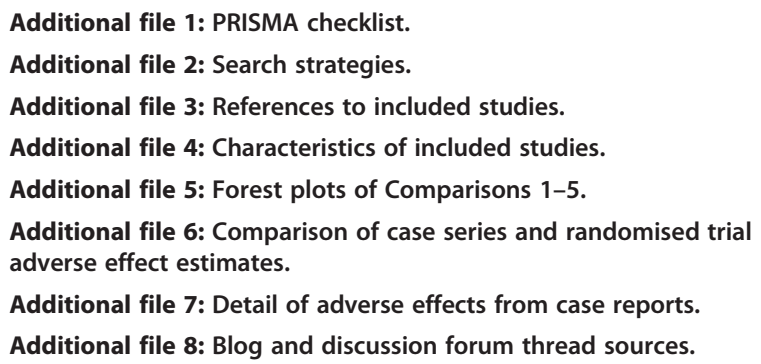




\section{Abbreviations}

Cl: Confidence interval; ICU: Intensive care unit; IM: Intramuscular; IV: Intravenous; OR: Odds ratio; RR: Risk ratio.

\section{Competing interests}

CAC was the principal investigator for the Australasian Collaborative Trial of Magnesium Sulphate. Two review authors (CAC and PFM) were co-authors of the Cochrane review 'Magnesium sulphate for women at risk of preterm birth for neuroprotection of the fetus'. The three review authors (CAC, ESB and PFM) were authors of the Cochrane review 'Different magnesium sulphate regimens for neuroprotection of the fetus'. CAC was an author of the Cochrane reviews 'Magnesium sulphate for preventing preterm birth in threatened preterm labour' and 'Magnesium maintenance therapy for preventing preterm birth after threatened preterm labour'.

\section{Authors' contributions}

ESB, CAC and PFM designed the study. ESB and PFM undertook searches, extracted and analysed data. ESB wrote the first draft of the paper. All authors supported the interpretation of results, provided comments on subsequent drafts and approved the final version.

\section{Author details}

${ }^{1}$ Australian Research Centre for Health of Women and Babies, Robinson Institute, Discipline of Obstetrics and Gynaecology, School of Paediatrics and Reproductive Health, The University of Adelaide, 72 King William Road, Adelaide, South Australia, Australia. 'Liggins Institute, The University of Auckland, Auckland, New Zealand.

Received: 11 June 2013 Accepted: 16 October 2013 Published: 21 October 2013

\section{References}

1. Duley L, Gülmezoglu A, Chou D: Magnesium sulphate versus lytic cocktail for eclampsia. Cochrane Database Syst Rev 2010, 9, CD002960.

2. Duley $L$, Henderson-Smart D, Chou D: Magnesium sulphate versus phenytoin for eclampsia. Cochrane Database Syst Rev 2010, 10, CD000128.

3. Duley L, Henderson-Smart D, Walker G, Chou D: Magnesium sulphate versus diazepam for eclampsia. Cochrane Database Syst Rev 2010, 12, CD000127.

4. Duley L, Gulmezoglu A, Henderson-Smart D, Chou D: Magnesium sulphate and other anticonvulsants for women with pre-eclampsia. Cochrane Database Syst Rev 2010, 11, CD000025.

5. Crowther CA, Hiller JE, Doyle LW: Magnesium sulphate for preventing preterm birth in threatened preterm labour. Cochrane Database Syst Rev 2002, 4, CD001060.

6. Han S, Crowther CA, Moore V: Magnesium maintenance therapy for preventing preterm birth after threatened preterm labour. Cochrane Database Syst Rev 2010, 7, CD000940.

7. Doyle LW, Crowther CA, Middleton P, Marret S, Rouse DJ: Magnesium sulphate for women at risk of preterm birth for neuroprotection of the fetus. Cochrane Database Syst Rev 2009, 1, CD004661.

8. Lu J, Nightingale C: Magnesium sulfate in eclampsia and pre-eclampsia: pharmacokinetic principles. Clin Pharmacokinet 2000, 38(4):305-314.

9. Duley L, Matar $\mathrm{H}$, Almerie M, Hall D: Alternative magnesium sulphate regimens for women with pre-eclampsia and eclampsia. Cochrane Database Syst Rev 2010, 8, CD007388.

10. Golder S, Loke YK, Bland M: Meta-analyses of adverse effects data derived from randomised controlled trials as compared to observational studies: methodological overview. PLoS Med 2011, 8(5):e1001026.

11. Papanikolaou PN, Christidi GD, Ioannidis JPA: Comparison of evidence on harms of medical interventions in randomized and nonrandomized studies. CMAJ 2006, 174(5):635-641.

12. Loke Y, Price D, Herxheimer A, Cochrane Adverse Effects Methods Group: Systematic reviews of adverse effects: framework for a structured approach. BMC Med Res Methodol 2007, 7:1-9.

13. Higgins J, Green S: Cochrane Handbook for Systematic Reviews of Interventions. Version 5.1.0. The Cochrane Collaboration; 2011. Available at: http://handbook.cochrane.org.

14. Wells G, Shea B, O'Connell D, Peterson J, Welch V, Losos M, Tugwell M: The Newcastle-Ottawa Scale (NOS) for assessing the quality of nonrandomised studies in meta-analyses. www.ohri.ca/programs/clinical_epidemiology/ oxford.htm.

15. Ioannidis JPA, Evans SJW, Gotzsche PC, O'Neill RT, Altman DG, Schulz K Moher D, CONSORT Group: Better reporting of harms in randomized trials: an extension of the CONSORT statement. Ann Intern Med 2004 141(1):781-788.

16. Chen FP, Chang SD, Chu KK: Expectant management in severe preeclampsia: does magnesium sulfate prevent the development of eclampsia? Acta Obstet Gynecol Scand 1995, 74:181-185.

17. Coetzee EJ, Dommisse J, Anthony J: A randomised controlled trial of intravenous magnesium sulphate versus placebo in the management of women with severe pre-eclampsia. Br J Obstet Gynaecol 1998, 105(3):300-303.

18. Cox SM, Sherman ML, Leveno KJ: Randomized investigation of magnesium sulfate for prevention of preterm birth. Am J Obstet Gynecol 1990, 163(3):767-772.

19. Crowther CA, Hiller JE, Doyle LW, Haslam RR, Australasian Collaborative Trial of Magnesium Sulphate Collaborative Group: Effect of magnesium sulfate given for neuroprotection before preterm birth: a randomized controlled trial. JAMA 2006, 290(2):2669-2676.

20. The Magpie Trial Collaborative Group: Do women with pre-eclampsia, and their babies, benefit from magnesium sulphate? The Magpie Trial: A randomised placebo-controlled trial. Lancet 2002, 359(9321):1877-1890.

21. Livingston JC, Livingston LW, Ramsey R, Mabie BC, Sibai BM: Magnesium sulfate in women with mild preeclampsia: a randomized controlled trial. Obstet Gynecol 2003, 101(2):217-220.

22. Ma L: Magnesium sulfate in prevention of preterm labor. Zhong hua yi xue za zhi 1992, 72(3):158-161.

23. Marret S, Marpeau L, Zupan-Simunek V, Eurin D, Leveque C, Hellot M-F, Benichou J, PREMAG trial group: Magnesium sulfate given before very-preterm birth to protect infant brain: the randomized controlled PREMAG trial. Br J Obstet Gynaecol 2007, 114(3):310-318.

24. Moodley J, Moodley W: Prophylactic anticonvulsant therapy in hypertensive crises of pregnancy the need for a large, randomized trial. Hypertens Pregnancy 1994, 13(3):245-252.

25. Rouse DJ, Hirtz DG, Thom E, Varner MW, Spong CY, Mercer BM, lams JD, Wapner RJ, Sorokin Y, Alexander JM, Harper M, Thorp JM, Ramin SM, Malone FD, Carpenter M, Miodovnik M, Moawad A, O'Sullivan MJ, Peaceman AM, Hankins GDV, Langer O, Caritis SN, Roberts JM, For the Eunice Kennedy Shiver NICHD Maternal-Fetal Medicine Units Network: A randomized, controlled trial of magnesium sulfate for the prevention of cerebral palsy. N Engl J Med 2008, 359:895-905.

26. Witlin AG, Friedman SA, Sibai BM: The effect of magnesium sulfate therapy on the duration of labor in women with mild preeclampsia at term: a randomized, double-blind, placebo-controlled trial. Am J Obstet Gynecol 1997, 176(3):623-627.

27. Shilva Saha SC, Kalra J, Prasad R: Safety and efficacy of low-dose MgSO4 in the treatment of eclampsia. Int J Gynaecol Obstet 2007, 97(2):150-151.

28. Malapaka SVN, Ballal PK: Low-dose magnesium sulfate versus Pritchard regimen for the treatment of eclampsia imminent eclampsia. Int J Gynaecol Obstet 2011, 115(1):70-72.

29. Bhattacharjee N, Saha SP, Ganguly RP, Patra KK, Dhali B, Das N, Barui G: A randomised comparative study between low-dose intravenous magnesium sulphate and standard intramuscular regimen for treatment of eclampsia. J Obstet Gynaecol 2011, 31(4):298-303.

30. Chissell S, Botha JH, Moodley J, McFadyen L: Intravenous and intramuscular magnesium sulphate regimens in severe pre-eclampsia. S Afr Med J 1994, 84(9):607-610.

31. Mundle S, Regi A, Biswas B, Bracken H, Easterling T, Winikoff B: Preeclampsia in low-resource settings: a randomized trial of IV MgSO4 via flow controlled pump. Int J Gynaecol Obstet 2009, 107(2):S278.

32. Ehrenberg HM, Mercer BM: Abbreviated postpartum magnesium sulfate therapy for women with mild preeclampsia: a randomized controlled trial. Obstet Gynecol 2006, 108(4):833-838.

33. Suneja A, Sinha S, Vaid N, Ahuja S: A prospective randomized controlled trial to individualize the duration of post partum magnesium sulfate therapy. Hypertens Pregnancy 2008, 27:504

34. Behrad V, Moossavifar N, Mojtahedzadeh M, Esmaili H, Moghtadeii P: A prospective, randomized, controlled trial of high and low doses of magnesium sulfate for acute tocolysis. Acta Med Iran 2003, 41(2):126-131.

35. Terrone DA, Rinehart BK, Kimmel ES, May WL, Larmon JE, Morrison JC: A prospective, randomized, controlled trial of high and low maintenance 
doses of magnesium sulfate for acute tocolysis. Am J Obstet Gynecol 2000, 182(6):1477-1482

36. Zygmunt M, Heilmann L, Berg C, Wallwiener D, Grischke E, Munstedt K, Spindler A, Lang U: Local and systemic tolerability of magnesium sulphate for tocolysis. Eur J Obstet Gynecol Reprod Biol 2003, 107(2):168-175.

37. Ales KL, Charlson ME: Prognosis of hypertension first documented during labor. Am J Perinatol 1987, 4(4):317-323.

38. Seyb ST, Berka RJ, Socol ML, Dooley SL: Risk of cesarean delivery with elective induction of labor at term in nulliparous women. Obstet Gynecol 1999, 94(4):600-607.

39. Park KH, Cho YK, Lee CM, Choi H, Kim BR, Lee HK: Effect of preeclampsia, magnesium sulfate prophylaxis, and maternal weight on labor induction: a retrospective analysis. Gynecol Obstet Invest 2006, 61(1):40-44

40. Assaley J, Baron JM, Cibils LA: Effects of magnesium sulfate infusion upon clotting parameters in patients with pre-eclampsia. J Perinat Med 1998, 26(2):115-119.

41. Kynczl-Leisure M, Cibils LA: Increased bleeding time after magnesium sulfate infusion. Am J Obstet Gynecol 1996, 175(5):1293-1294.

42. Ramanathan J, Sibai BM, Duggirala V, Maduska AL: Pulmonary function in preeclamptic women receiving MgSO4. J Reprod Med 1988, 33(5):432-435.

43. Ramanathan J, Sibai BM, Pillai R, Angel JJ: Neuromuscular transmission studies in preeclamptic women receiving magnesium sulfate. Am J Obstet Gynecol 1988, 158(1):40-46.

44. Poggi SH, Barr S, Cannum R, Collea JV, Landy HJ, Kezsler M, Ghidini A: Risk factors for pulmonary edema in triplet pregnancies. J Perinatol 2003 23(6):462-465

45. Magee LA, Miremadi S, Li J, Cheng C, Ensom MHH, Carleton B, Cote A-M, Von-Dadelszan P: Therapy with both magnesium sulfate and nifedipine does not increase the risk of serious magnesium-related maternal side effects in women with preeclampsia. Am J Obstet Gynecol 2005, 193(1):153-163.

46. Chowdhury JR, Chaudhuri S, Bhattacharyya N, Biswas PK, Panpalia M: Comparison of intramuscular magnesium sulfate with low dose intravenous magnesium sulfate regimen for treatment of eclampsia. J Obstet Gynaecol Res 2009, 35(1):119-125.

47. Mahajan NN, Thomas A, Soni RN, Gaikwad NL, Jain SM: 'Padhar regime' - a low-dose magnesium sulphate treatment for eclampsia. Gynecol Obstet Invest 2009, 67(1):20-24.

48. Young BK, Weinstein HM: Effects of magnesium sulfate on toxemic patients in labor. Obstet Gynecol 1977, 49(6):681-685.

49. Shoaib T, Khan S, Javed I, Bhutta SZ: Loading dose of magnesium sulphate versus standard regime for prophylaxis of pre-eclampsia. J Coll Physicians Surg Pak 2009, 19(1):30-33.

50. Palmer L, Newby BD: Development of a simplified protocol for administration of $20 \%$ magnesium sulphate for prophylaxis and treatment of eclampsia. Can J Hosp Pharm 2009, 62(6):490-495.

51. Nassar AH, Sakhel K, Maarouf $H$, Naassan GR, Usta IM: Adverse maternal and neonatal outcome of prolonged course of magnesium sulfate tocolysis. Acta Obstet Gyneco/ Scand 2006, 85(9):1099-1103.

52. Aali BS, Khazaeli $P$, Ghasemi F: Ionized and total magnesium concentration in patients with severe preeclampsia-eclampsia undergoing magnesium sulfate therapy. J Obstet Gynaecol Res 2007, 33(2):138-143.

53. Adewole IF, Oladokun A, Okewole Al, Omigbodun AO, Afolabi A, Ekele B, Audu LR, Obed $Y$ : Magnesium sulphate for treatment of eclampsia: the Nigerian experience. Afr J Med Med Sci 2000, 29(3-4):239-241.

54. Ekele BA, Badung SLH: Is serum magnesium estimate necessary in patients with eclampsia on magnesium sulphate? Afr J Reprod Health 2005, 9(1):128-132

55. Pritchard JA, Cunningham FG, Pritchard SA: The Parkland Memorial Hospital protocol for treatment of eclampsia: evaluation of 245 cases Am J Obstet Gynecol 1984, 148(7):951-963.

56. Raman NV, Rao CA: Magnesium sulfate as an anticonvulsant in eclampsia. Int J Gynaecol Obstet 1995, 49(3):289-298.

57. Elliott JP: Magnesium sulfate as a tocolytic agent. Am J Obstet Gynecol 1983, 147(3):277-284

58. Girard B, Beucher G, Muris C, Simonet T, Dreyfus M: Magnesium sulphate and severe preeclampsia: its use in current practice. J Gynecol Obstet Biol Reprod 2005, 34(1 Pt 1):17-22

59. Harding $K$, Erskine $K$, Howell $P$ : Audit of the impact of magnesium sulphate on the management of severe pre-eclampsia in a London university hospital. Eur J Anaesthesiol 1997, 14(5):527-528.
60. Thapa K, Jha R: Magnesium sulphate: a life saving drug. J Nepal Med Assoc 2008, 47(171):104-108.

61. Dasari $P$, Habeebullah S: Maternal mortality due to hypertensive disorders of pregnancy in a tertiary care center in Southern India. Int J Gynaecol Obstet 2010, 110(3):271-273.

62. Donovan EF, Tsang RC, Steichen JJ, Strub RJ, Chen IW, Chen M: Neonatal hypermagnesemia: effect on parathyroid hormone and calcium homeostasis. J Pediatr 1980, 96(2):305-310.

63. Mojadidi Q, Thompson RJ: Five years' experience with eclampsia. South Med 1973, 66(4):414-416.

64. Tukur J, Muhammad Z: Management of eclampsia at AKTH: before and after magnesium sulphate. Niger J Med 2010, 19(1):104-107.

65. Getaneh W, Kumbi S: Use of magnesium sulfate in pre-eclampsia and eclampsia in teaching hospitals in Addis Ababa: a practice audit. Ethiop Med J 2010, 48(2):157-164.

66. Ahmed R: Magnesium sulphate as an anticonvulsant in the management of eclampsia. J Coll Physicians Surg Pak 2004, 14(10):605-607.

67. Begum R, Begum A, Johanson R, Ali MN, Akhter S: A low dose ("Dhaka") magnesium sulphate regime for eclampsia. Acta Obstet Gynecol Scand 2001, 80(11):998-1002.

68. Digre KB, Varner MW, Schiffman JS: Neuroophthalmologic effects of intravenous magnesium sulfate. Am J Obstet Gynecol 1990, 163(6 Pt 1):1848-1852

69. Hales KA, Matthews JP, Rayburn WF, Atkinson BD: Intravenous magnesium sulfate for premature labor: comparison between twin and singleton gestations. Am J Perinatol 1995, 12(1):7-10.

70. Sass N, Mesquita M, Kenji G, Goncalves F, Arauj F: Do women with preeclampsia benefit from an exclusively intravenous magnesium sulphate regimen? Is 2.0 grams/hour effective? (abstract). Hypertens Pregnancy 2007, 4:\$591.

71. Omu AE, Al-Harmi J, Vedi HL, Mlechkova L, Sayed AF, Al-Ragum NS: Magnesium sulphate therapy in women with pre-eclampsia and eclampsia in Kuwait. Med Princ Pract 2008, 17(3):227-232

72. Cotton DB, Gonik B, Dorman KF: Cardiovascular alterations in severe pregnancy-induced hypertension: acute effects of intravenous magnesium sulfate. Am J Obstet Gynecol 1984, 148(2):162-165.

73. Yeast JD, Halberstadt C, Meyer BA, Cohen GR, Thorp JA: The risk of pulmonary edema and colloid osmotic pressure changes during magnesium sulfate infusion. Am J Obstet Gynecol 1993, 169(6):1566-1571.

74. Jirapinyo M, Thuvasethakul $P$, Leelaphiwat S: Prospective study on premature labor with magnesium sulfate. Asia Oceania J Obstet Gynaecol 1990, 16(2):91-96.

75. Kfuri TA, Morlock L, Hicks RW, Shore AD: Medication errors in obstetrics. Clin Perinatol 2008, 35(1):101-117.

76. Little JA, Velazquez MB, Rayburn WF: Reported medication errors in obstetric inpatients in 1 hospital. J Reprod Med 2003, 48(10):818-820.

77. Bilgin T, Cengiz C, Ozan H: Alterations in respiratory functions during magnesium sulfate infusion in severe preeclampsia and eclampsia. Int $J$ Gynaecol Obstet 1994, 45(1):59-60.

78. Herpolsheimer A, Brady K, Yancey MK, Pandian M, Duff P: Pulmonary function of preeclamptic women receiving intravenous magnesium sulfate seizure prophylaxis. Obstet Gynecol 1991, 78(2):241-244.

79. Ghia N, Spong CY, Starbuck VN, Scialli AR, Ghidini A: Magnesium sulfate therapy affects attention and working memory in patients undergoing preterm labor. Am J Obstet Gynecol 2000, 183(4):940-944.

80. Fuentes A, Rojas A, Porter KB, Saviello G, O'Brien WF: The effect of magnesium sulfate on bleeding time in pregnancy. Am J Obstet Gynecol 1995, 173(4):1246-1249.

81. Guzin K, Goynumer G, Gokdagli F, Turkgeldi E, Gunduz G, Kayabasoglu F: The effect of magnesium sulfate treatment on blood biochemistry and bleeding time in patients with severe preeclampsia. J Matern Fetal Neonatal Med 2010, 23(5):399-402.

82. Yazdani M, Jahvani FA, Tabei SZ: Effects of magnesium sulfate on bleeding time in premature labor. Iran J Med Sci 2004, 29(4):172-174.

83. Moghadas FRS, Motevalian M, Rezai Z, Ehteshamipour E: The effect of magnesium sulfate therapy on bleeding time in women with threatened preterm labor. Iran J Pharmacol Ther 2007, 6(1):55-57.

84. Anon: Magnesium overdose kills pregnant R.Ph.; her twins saved. Drug Top 1990, 134:11-12

85. Cohen MR, Davis NM: Free flow associated with electronic infusion devices: underestimated danger. Hosp Pharm 1992, 27(5):384-390. 
86. Richards A, Stather-Dunn L, Moodley J: Cardiopulmonary arrest after the administration of magnesium sulphate. A case report. S Afr Med J 1985, 67(4):145

87. Simpson KR, Knox GE: Obstetrical accidents involving intravenous magnesium sulfate: recommendations to promote patient safety. MCN Am J Matern Child Nurs 2004, 29(3):161-169.

88. McCubbin JM, Sibai BM, Ardella TN, Anderson GD: Cardiopulmonary arrest due to acute maternal hypermagnesaemia. Lancet 1981, 1(8228):1058.

89. McDonnell NJ: Cardiopulmonary arrest in pregnancy: two case reports of successful outcomes in association with perimortem Caesarean delivery. Br J Anaesth 2009, 103(3):406-409.

90. Morisaki H, Yamamoto S, Morita Y, Kotake Y, Ochiai R, Takeda J: Hypermagnesemia-induced cardiopulmonary arrest before induction of anesthesia for emergency cesarean section. J Clin Anesth 2000, 12(3):224-226.

91. Rabinerson D, Gruber A, Kaplan B, Royburt M, Ovadia J: Accidental cardiopulmonary arrest following magnesium sulphate overdose. Eur J Obstet Gynecol Reprod Biol 1994, 55(2):149-150.

92. Swartjes JM, Schutte MF, Bleker OP: Management of eclampsia: cardiopulmonary arrest resulting from magnesium sulfate overdose. Eur J Obstet Gynecol Reprod Biol 1992, 47(1):73-75.

93. Bohman VR, Cotton DB: Supralethal magnesemia with patient survival. Obstet Gynecol 1990, 76(5 Pt 2):984-986.

94. Cao Z, Bideau R, Valdes R Jr, Elin RJ: Acute hypermagnesemia and respiratory arrest following infusion of $\mathrm{MgSO} 4$ for tocolysis. Clin Chim Acta 1999, 285(1-2):191-193.

95. McKenna D: That's our drip counter! Lancet 1873, 2006:367(9525).

96. Wax JR, Segna RA, Vandersloot JA: Magnesium toxicity and resuscitationan unusual cause of postcesarean evisceration. Int J Gynaecol Obstet 1995, 48(2):213-214

97. Bruhwiler $H$, Hafliger M, Luscher KP: Severe accidental magnesium poisoning in a twins pregnancy in the 32 nd week of pregnancy. Geburtshilfe Frauenheilkd 1994, 54(3):184-186.

98. Hayashi K, Oshiro M, Takara I, Iha H, Sugahara K: Coma caused by hypermagnesemia in a pregnant woman complicated with HELLP syndrome. Masui 2003, 52(7):783-785

99. McDonnell NJ, Muchatuta NA, Paech MJ: Acute magnesium toxicity in an obstetric patient undergoing general anaesthesia for caesarean delivery. Int J Obstet Anesth 2010, 19(2):226-231.

100. Buettner AU: Two cases of inadvertent magnesium sulphate overdose. Int J Obstet Anesth 2011, 20(1):92-93.

101. Dror A, Henriksen E: Accidental epidural magnesium sulfate injection. Anesth Analg 1987, 66(10):1020-1021.

102. Goodman EJ, Haas AJ, Kantor GS: Inadvertent administration of magnesium sulfate through the epidural catheter: report and analysis of a drug error. Int J Obstet Anesth 2006, 15(1):63-67.

103. Lejuste MJ: Inadvertant intrathecal administration of magnesium sulfate. S Afr Med J 1985, 68(6):367-368

104. Lewis-Younger C, Speranza V, Gaar G: Temporary paralysis resulting from medical error. J Toxicol Clin Toxicol 2004, 42(5):732.

105. Bashuk RG, Krendel DA: Myasthenia gravis presenting as weakness after magnesium administration. Muscle Nerve 1990, 13(8):708-712.

106. Bruner JP, Yeast JD: Pregnancy associated with Friedreich ataxia. Obstet Gynecol 1990, 76(5 Pt 2):976-977.

107. Catanzarite V, Gambling D, Bird LM, Honold J, Perkins E: Respiratory compromise after MgSO4 therapy for preterm labor in a woman with myotonic dystrophy: a case report. J Reprod Med 2008, 53(3):220-222.

108. Hosono T, Suzuki M, Chiba Y: Contraindication of magnesium sulfate in a pregnancy complicated with late-onset diabetes mellitus and sensory deafness due to mitochondrial myopathy. J Matern Fetal Med 2001, 10:355-356

109. Cohen BA, London RS, Goldstein PJ: Myasthenia gravis and preeclampsia. Obstet Gynecol 1976, 48(1 Suppl):35S-37S.

110. Mueksch JN, Stevens WA: Undiagnosed myasthenia gravis masquerading as eclampsia. Int J Obstet Anesth 2007, 16(4):379-382.

111. Robins K, Lyons G: Opioid-related narcosis in a woman with myopathy receiving magnesium. Int J Obstet Anesth 2007, 16(4):367-369.

112. Moriarty KT, McFarland R, Whittaker R, Burch J, Turnbull HE, Taylor RW, Turnbull DM: Pre-eclampsia and magnesium toxicity with therapeutic plasma level in a woman with m.3243A > G melas mutation. J Obstet Gynaecol 2008, 28(3):349
113. Archer TL, Heitmeyer JD: Perioperative hemodynamics obtained by pulse contour analysis facilitated the management of a patient with chronic hypertension, renal insufficiency, and superimposed preeclampsia during cesarean delivery. J Clin Anesth 2010, 22(4):274-279.

114. Chan SM, Lu CC, Ho ST, Liaw WJ, Cherng CH, Chen WH, Lin TC: Eclampsia following cesarean section with HELLP syndrome and multiple organ failure. Acta Anaesthesiol Taiwan 2008, 46(1):46-48.

115. Nethravathi M, Panicker JN, Taly AB, Arunodaya GR, Sinha S: Therapeutic magnesium for eclampsia: an unusual cause for antepartum flaccid paralysis. J Postgrad Med 2007, 53(1):79-80.

116. Saitoh K, Motegi R, Hirabayashi Y, Shimizu R: Cardiac arrests probably induced by hypermagnesemia during anesthesia for caesarean section. Masui 1994, 43(3):388-391.

117. Baraka A, Dajani A, Jabagi S: Magnesium-induced respiratory arrest in a parturient recovering from general anesthesia-a case report. Middle East $J$ Anesthesiol 1984, 7(6):437-440.

118. Nguyen TTT, Koenders MEF, Wierda JMKH: Drug interaction in a pregnancy complicated by pre-eclampsia and cesarean section. Ned Tijdschr Anesthesiol 2001, 14(1):21-25.

119. Fay TN, Bogod D: Magnesium sulphate: the time for reckoning. Br J Obstet Gynaecol 1996, 103(8):852.

120. Funai $Y$, Ikeda $Y$, Takahashi $R$, Asada A: Prolonged apnea from rocuronium in a patient with hypermagnesemia after cesarean section: a case report. Masui 2010, 59(6):721-723.

121. Hino H, Kaneko I, Miyazawa A, Aoki T, Ishizuka B, Kosuqi K, Amemiya A: Prolonged neuromuscular blockade with vecuronium in patient with triple pregnancy treated with magnesium sulfate. Masui 1997, 46(2):266-270.

122. Kwan WF, Lee $C$, Chen BJ: A noninvasive method in the differential diagnosis of vecuronium-induced and magnesium-induced protracted neuromuscular block in a severely preeclamptic patient. $J$ Clin Anesth 1996, 8(5):392-397.

123. Sinatra RS, Philip BK, Naulty JS, Ostheimer GW: Prolonged neuromuscular blockade with vecuronium in a patient treated with magnesium sulfate. Anesth Analg 1985, 64(12):1220-1222.

124. Yoshida A, Itoh Y, Nagaya K, Takino K, Sugawara J-I, Murakami T, Okamura K, Takahashi M: Prolonged relaxant effects of vecuronium in patients with deliberate hypermagnesemia: time for caution in cesarean section. J Anesth 2006, 20(1):33-35.

125. Sloan PA, Rasul M: Prolongation of rapacuronium neuromuscular blockade by clindamycin and magnesium. Anesth Analg 2002, 94(1):123-124.

126. Ben-Ami M, Giladi Y, Shalev E: The combination of magnesium sulphate and nifedipine: a cause of neuromuscular blockade. Br J Obstet Gynaecol 1994, 101(3):262-263

127. Snyder SW, Cardwell MS: Neuromuscular blockade with magnesium sulfate and nifedipine. Am J Obstet Gynecol 1989, 161(1):35-36.

128. Wu Q-A, Ye Y-Q: Neuromuscular blockade after therapy with magnesium sulfate and amlodipine. Eur J Obstet Gynecol Reprod Biol 2010, 149(2):225.

129. Pittman JA: Magnesium sulphate for pre-eclampsia and a sudden bradycardia. Br J Anaesth 2000, 85(2):327-328.

130. Scardo JA, Brost BC, Sola E, Newman RB, Tate SB: Severe hypotension with nifedipine-magnesium in severe preeclampsia: $A$ hemodynamic observation. J Matern Fetal Investig 1997, 7(3):152-154.

131. Waisman GD, Mayorga LM, Camera MI, Vignolo CA, Martinotti A: Magnesium plus nifedipine: potentiation of hypotensive effect in preeclampsia? Am J Obstet Gynecol 1988, 159(2):308-309.

132. Awwad JT, Khalil AM, Aswad NK, Suidan FJ, Karam KS: Labial edema in pregnancy. A case report. J Reprod Med 1994, 39(11):921-922.

133. Basaran A, Bozdag G, Aksu AT, Deren O: Twin pregnancy complicated with acute appendicitis and cholecystitis in the same gestational period. Arch Gynecol Obstet 2007, 276(3):291-293.

134. Haldeman W: Can magnesium sulfate therapy impact lactogenesis? J Hum Lact 1993, 9(4):249-252.

135. Hill WC, Gill PJ, Katz M: Maternal paralytic ileus as a complication of magnesium sulfate tocolysis. Am J Perinatol 1985, 2(1):47-48.

136. Hung J-W, Tsai M-Y, Yang B-Y, Chen J-F: Maternal osteoporosis after prolonged magnesium sulfate tocolysis therapy: a case report. Arch Phys Med Rehabil 2005, 86(1):146-149.

137. Lurie S, Rotmensch S, Feldman N, Glezerman M: Breast engorgement and galactorrhea during magnesium sulfate treatment of preterm labor. Am Perinatol 2002, 19(5):239-240. 
138. Riggs JE, Hogg JP: Central pontine myelinolysis: association with parenteral magnesium administration. Mil Med 2000, 165(6):494-495

139. Sameshima H, Higo T, Kodama Y, Ikenoue T: Magnesium tocolysis as the cause of urinary calculus during pregnancy. J Matern Fetal Med 1997, 6(5):296-297.

140. Spital A, Greenwell R: Severe hyperkalemia during magnesium sulfate therapy in two pregnant drug abusers. South Med J 1991, 84(7):919-921.

141. Roberts D, Haslett E, Hickey-Dwyer M, McCormack J: Eclampsia complicated by bilateral retinal detachments and abnormal eye movements. Lancet 1998, 351(9105):803-804.

142. Thorp JM Jr, Katz VL, Campbell D, Cefalo RC: Hypersensitivity to magnesium sulfate. Am J Obstet Gynecol 1989, 161(4):889-890.

143. Bourgeois FJ, Thiagarajah S, Harbert GM Jr, DiFazio C: Profound hypotension complicating magnesium therapy. Am J Obstet Gynecol 1986, 154(4):919-920.

144. Rodis JF, Vintzileos AM, Campbell WA, Deaton JL, Nochimson DJ: Maternal hypothermia: an unusual complication of magnesium sulfate therapy. Am J Obstet Gynecol 1987, 156(2):435-436.

145. Cardosi RJ, Chez RA: Magnesium sulfate, maternal hypothermia, and fetal bradycardia with loss of heart rate variability. Obstet Gynecol 1998, 92(4 Pt 2):691-693.

146. Hennessy A, Hill I: A case of maternal bradycardia at therapeutic doses of magnesium sulphate in preeclampsia. Aust N Z J Obstet Gynaecol 1999, 39(2):256-257.

147. Oettinger M, Perlitz Y: Asymptomatic paroxysmal atrial fibrillation during intravenous magnesium sulfate treatment in preeclampsia. Gynecol Obstet Invest 1993, 36(4):244-246.

148. Pritchard JA: The use of magnesium sulfate in preeclampsia-eclampsia. J Reprod Med 1979, 23(3):107-114.

149. Herschel M, Mittendorf R: Tocolytic magnesium sulfate toxicity and unexpected neonatal death. J Perinatol 2001, 21(4):261-262.

150. Tang F, Xiao B, Xiong Q, Yang M: A case report: magnesium intoxication occurring in the process of total serum magnesium decrease. J Obstet Gynaecol Res 2010, 36(1):174-177.

151. Sherer D, Cialone P, Abramowicz J, Woods J: Transient symptomatic subendocardial ischemia suring magnesium sulfate tocolytic therapy. Am J Obstet Gynecol 1992, 166(1 Pt 1):33-35

152. Koontz SL, Friedman SA, Schwartz ML: Symptomatic hypocalcemia after tocolytic therapy with magnesium sulfate and nifedipine. Am J Obstet Gynecol 2004, 190(6):1773-1776.

153. Mayan H, Hourvitz A, Schiff E, Farfel Z: Symptomatic hypocalcaemia in hypermagnesaemia-induced hypoparathyroidism, during magnesium tocolytic therapy - Possible involvement of the calcium-sensing receptor. Nephrol Dial Transplant 1999, 14(7):1764-1766.

154. Monif GR, Savory J: latrogenic maternal hypocalcemia following magnesium sulfate therapy. JAMA 1972, 219(11):1469-1470.

155. Nassar AH, Salti I, Makarem NN, Usta IM: Marked hypocalcemia after tocolytic magnesium sulphate therapy. Am J Perinatol 2007, 24(8):481-482.

156. Ganzevoort JW, Hoogerwaard EM, van der Post JAM: Hypocalcemic delirium due to magnesium sulphate therapy in a pregnant woman with pre-eclampsia]. Ned Tijdschr Geneeskd 2002, 146(31):1453-1456.

157. Elliott JP, O'Keeffe DF, Greenberg P, Freeman RK: Pulmonary edema associated with magnesium sulfate and betamethasone administration. Am J Obstet Gynecol 1979, 134(6):717-719.

158. Worrell JA, Brunner JP, O'Donnell DM, Carroll FE: Pulmonary edema associated with tocolytic therapy. AJR Am J Roentgenol 1992, 158(6):1356-1357.

159. Institute for Safe Medical Practices: ISMP Medication Safety Alert. Enhance systems to ensure safe IV magnesium use. http://www.ismp.org/Newsletters/ acutecare/articles/19970212.asp.

160. Office of Safety \& Quality in Healthcare Government of Western Australia: Sharing Lessons Learned - Feb 2010. Preventing Magnesium Sulphate Overdose in Obstetric Settings. http://www.safetyandquality.health.wa.gov.au/home/ patient_news.cfm

161. Duley L: The global impact of pre-eclampsia and eclampsia. Semin Perinatol 2009, 33(3):130-137.

162. Tucker J, McGuire W: Epidemiology of preterm birth. BMJ 2004, 329:675-678

163. Loke YK, Golder SP, Vandenbroucke JP: Comprehensive evaluations of the adverse effects of drugs: importance of appropriate study selection and data sources. Ther Adv Drug Saf 2011, 2(2):59-68.
164. Kremer $L$, Phillips $R$, Van-Dalen E: Tips and tricks for understanding and using SR results - no 13: adverse effects in systematic reviews. Evidence-Based Child Health 2009, 4:384-385.

165. Grimes D, Nanda K: Magnesium sulfate tocolysis: time to quit. Obstet Gynecol 2006, 108(4):986-989.

166. U.S. Food and Drug Administration: FDA recommends against prolonged use of magnesium sulfate to stop pre-term labor due to bone changes in exposed babies. http://www.fda.gov/Drugs/DrugSafety/ucm353333.htm.

167. loannidis JPA: Adverse events in randomized trials: neglected, restricted, distorted, and silenced. Arch Intern Med 2009, 169(19):1737-1739.

doi:10.1186/1471-2393-13-195

Cite this article as: Bain et al:: Maternal adverse effects of different antenatal magnesium sulphate regimens for improving maternal and infant outcomes: a systematic review. BMC Pregnancy and Childbirth 2013 13:195

\section{Submit your next manuscript to BioMed Central and take full advantage of:}

- Convenient online submission

- Thorough peer review

- No space constraints or color figure charges

- Immediate publication on acceptance

- Inclusion in PubMed, CAS, Scopus and Google Scholar

- Research which is freely available for redistribution 